\title{
The VISTA Orion mini-survey: star formation in the Lynds 1630 North cloud $\star, \star \star$
}

\author{
L. Spezzi ${ }^{1,2}$, M. G. Petr-Gotzens ${ }^{1}$, J. M. Alcalá3 ${ }^{3}$ J. K. Jørgensen ${ }^{4}$, T. Stanke ${ }^{1}$, M. Lombardi ${ }^{5,6}$, and J. F. Alves ${ }^{7}$ \\ ${ }^{1}$ European Southern Observatory, Karl-Schwarzschild-Straße 2, 85748 Garching bei München, Germany \\ e-mail: loredana.spezzi@gmail.com \\ ${ }^{2}$ European Organisation for the Exploitation of Meteorological Satellites (EUMETSAT), Eumetsat Allee 1, 64295 Darmstadt, \\ Germany \\ 3 INAF-Osservatorio Astronomico di Capodimonte, via Moiariello, 16, 80131 Napoli, Italy \\ ${ }^{4}$ Niels Bohr Institute, University of Copenhagen, Juliane Maries Vej 30, 2100 Copenhagen Ø, Denmark \\ 5 University of Milan, Department of Physics, via Celoria 16, 20133 Milan, Italy \\ ${ }^{6}$ Harvard-Smithsonian Center for Astrophysics, Mail Stop 72, 60 Garden Street, Cambridge, MA 02138, USA \\ 7 Institute for Astronomy, University of Vienna, Türkenschanzstr. 17, 1180 Vienna, Austria
}

Received 26 November 2014 / Accepted 16 May 2015

\section{ABSTRACT}

\begin{abstract}
The Orion cloud complex presents a variety of star formation mechanisms and properties and is still one of the most intriguing targets for star formation studies. We present VISTA/VIRCAM near-infrared observations of the L1630N star-forming region, including the stellar clusters NGC 2068 and NGC 2071 in the Orion molecular cloud B, and discuss them in combination with Spitzer data. We select 186 young stellar object (YSO) candidates in the region on the basis of multi-color criteria, confirm the YSO nature of the majority of them using published spectroscopy from the literature, and use this sample to investigate the overall star formation properties in L1630N. The $K$-band luminosity function of $\mathrm{L} 1630 \mathrm{~N}$ is remarkably similar to that of the Trapezium cluster, i.e., it presents a broad peak in the range $0.3-0.7 M_{\odot}$ and a fraction of substellar objects of $\sim 20 \%$. The fraction of YSOs still surrounded by disk/envelopes is very high ( $\sim 85 \%)$ compared to other star-forming regions of similar age (1-2 Myr), but includes some uncertain corrections for diskless YSOs. Yet, a possibly high disk fraction, together with the fact that $1 / 3$ of the cloud mass has a gas surface density above the threshold for star formation $\left(\sim 129 \mathrm{M}_{\odot} \mathrm{pc}^{-2}\right)$, points toward a still ongoing star formation activity in L1630N. The star formation efficiency (SFE), star formation rate (SFR), and density of star formation of L1630N are within the ranges estimated for Galactic star-forming regions by the Spitzer core to disk and Gould's Belt surveys. However, the SFE and SFR are lower than the average value measured in the Orion A cloud and, in particular, lower than that in the southern regions of L1630. This might suggest different star formation mechanisms within the L1630 cloud complex.
\end{abstract}

Key words. surveys - stars: pre-main sequence - open clusters and associations: individual: L1630N - stars: formation stars: protostars - ISM: clouds

\section{Introduction}

Observations of young stellar populations in nearby star-forming regions are important tools to understand the interplay between the outcome of the star formation process and the original environment from which the stellar ensembles emerged. While details of the star formation process and its physics are often tested with targeted investigations on small spatial scales, global properties are best assessed with wide-field imaging surveys in the infrared thereby accomplishing large-scale studies in a homogenous way.

The Spitzer c2d (Evans et al. 2009) and Spitzer Gould Belt (GB) ${ }^{1}$ Legacy surveys (e.g., Spezzi et al. 2011; Hatchell et al. 2012; Dunham et al. 2013) effectively traced the population of young stellar objects (YSOs) in several nearby star-forming regions. These studies have shown that current star formation

\footnotetext{
* Based on observations collected at the ESO La Silla Paranal Observatory under programme ID 060.A-9285(B).

$\star \star$ Tables A.1 and A.2 are are only available at the CDS via anonymous ftp to cdsarc.u-strasbg. fr (130.79.128.5) or via http://cdsarc.u-strasbg.fr/viz-bin/qcat?J/A+A/581/A140 1 http://www.cfa.harvard.edu/gouldbelt
}

efficiencies are in the range from $3 \%$ to $6 \%$, and that star formation is highly concentrated to regions of high extinction with the youngest objects being strongly associated with dense cores. The great majority (90\%) of the young stars lie within loose clusters with at least 35 members and a stellar density of $1 M_{\odot} \mathrm{pc}^{-3}$ (Evans et al. 2009, and references therein). The c2d and GB surveys have also shown that the star formation surface density in Galactic star-forming regions is more than an order of magnitude larger than predicted from the extragalactic relationship between the star formation rate and gas density, e.g., the KennicuttSchmidt law (Evans et al. 2009; Heiderman et al. 2010).

Among the most-studied nearby active star formation sites are the Orion A and Orion B molecular clouds. The clouds have similar masses of a few $10^{4} M_{\odot}$ and appear physically connected, indicating that they stem from the same overall giant molecular cloud complex. However, star formation differs quite significantly between the clouds. In Orion B almost all stars $(\sim 90 \%)$ formed in stellar clusters (Lada et al. 1991), which concentrate at two major sites, one in the southern part of the Orion B cloud, where the clusters NGC 2024/23 are located, and one in the northern part of Orion B (also named L1630N) with the clusters NGC 2068/71. Orion A, on the other hand, shows a 
substantial population of distributed star formation with $\sim 70 \%$ of the stars forming in isolation (Strom et al. 1993; Fang et al. 2009), with the exception of the Orion Nebula Cluster (ONC), which lies at the northernmost end of Orion A. Orion B contains several early B-type stars and at least one O star, while Orion A (excluding the ONC) possibly has no stars earlier than B4 and is apparently deficient in early type massive stars when compared to its known numbers of low-mass stars (Hsu et al. 2012). Furthermore, large-scale molecular gas maps indicate clear substructure on scales $<2$ pc in Orion A, whereas Orion B displays very little substructure, although highly filamentary molecular gas seems associated with the star-forming regions in the northern part of Orion B, i.e., in L1630 N (Gibb 2008, and references therein).

In this paper we present multiband wide-field nearinfrared observations obtained with VISTA/VIRCAM, combined with mid-infrared data from Spitzer, covering approximately 1.6 sq. deg in the northern part of the Orion molecular cloud B, i.e., in L1630 N.

The $\mathrm{L} 1630 \mathrm{~N}$ region contains the prominent bright optical reflection nebulosities NGC 2068 and NGC 2071 which, observed at near-infrared wavelengths, reveal their full nature as young stellar clusters. Flaherty \& Muzerolle (2008) determined the clusters' age as 1-2 Myr, depending on the models used, and confirmed 67 stellar members through optical spectroscopy. However, the use of optical spectroscopy, combined with 2MASS photometry, limited their study to the least embedded and slightly higher mass objects. Fang et al. (2009) also performed optical spectroscopy of 132 stars in the region. This latter sample includes all the stars previously characterized by Flaherty \& Muzerolle (2008) and several additional objects classified as pre-main sequence (PMS) stars by Fang et al. (2009). These authors find a much higher disk frequency in L1630 N in comparison with L1641 (Orion A) and with other star-forming regions of similar age, like Chamaeleon I and IC 348, but they also caution that the results are upper limits as their sample is biased against nondisk-bearing young stars. A recent study by Hsu et al. (2012) employed a photometric and spectroscopic survey to enlarge the population of confirmed members in L1641 and find the disk frequency practically as high as in L1630. Clearly, the physical characterization of young star properties becomes better defined as our census of the young star populations becomes complete.

The combination of wide field coverage and excellent sensitivity of our survey enables us to uncover a large young stellar object (YSO) population in L1630 N, which increases the number of previously known YSOs by a factor 1.5, and thereby allows us to analyze the global star formation properties in this region.

After the description of the VISTA-Orion catalog and the extraction of the data for this work in Sect. 2, we present the selection procedure of the YSO candidates in L1630 N in Sect. 3. Then, in Sects. 4 and 5 we investigate the $K$-band luminosity function, initial mass function, and proto-planetary disk fraction for the identified sample of YSO candidate members in L1630 N, as well as compare our results to other nearby young stellar clusters and associations. We study the spatial distribution and clustering properties of YSOs and independently confirm the known stellar clusters NGC 2068 and NGC 2071, but also identify a new stellar group around the Herbig-Haro objects HH24-26 (Sect. 6). In combination with an extinction map, derived from the same VISTA data, we also present the results on the global properties of star formation in the region and in the identified substructures (Sect. 7). Our conclusions are presented in Sect. 8.

\section{Observations and data reduction}

\subsection{VISTA data reduction and catalog extraction}

The Visible and Infrared Survey Telescope for Astronomy (VISTA) located at ESO Paranal Observatory is a $4 \mathrm{~m}$ class telescope equipped with a near-IR camera (VIRCAM) containing 16 detectors, for a total FoV of $1^{\circ} \times 1.5^{\circ}$ and a pixel scale of $0.339^{\prime \prime} /$ pix, and available broad- and narrowband filters in the wavelength range 0.9-2.2 $\mu \mathrm{m}$ (Emerson et al. 2006; Dalton et al. 2006). Data for L $1630 \mathrm{~N}$ were taken during the VISTA Science Verification (SV) as part of the program "VISTA SV Galactic Mini-survey in Orion" (PI: M. Petr-Gotzens; Petr-Gotzens et al. 2011). This survey consists of $Z Y J H K_{\mathrm{S}}$ images obtained during 14 nights between 16 October and 2 November 2009. The survey area is a mosaic of 20 VISTA fields with each field containing 6 pointings that are mosaicked together to form a socalled filled tile. The total survey covers $\sim 30 \mathrm{sq}$. deg around the Orion Belt stars. The region L1630 N is located in the VISTA Orion survey tile No. 12 roughly centered at RA $=05^{\mathrm{h}} 46^{\mathrm{m}} 41^{\mathrm{s}}$, Dec $=+00^{\mathrm{d}} 09^{\prime} 00^{\prime \prime}$ (Fig. 1), and contains the young stellar clusters NGC 2068 and NGC 2071, which clearly stand out on the VISTA near-infrared image (Fig. 2). Further details on the observing strategy, the exposure times per filter and particular observing patterns chosen for the VISTA Mini-survey in Orion were described in Arnaboldi et al. (2010) and Petr-Gotzens et al. (2011).

The data reduction was performed by a dedicated pipeline, developed within the VISTA Data Flow System (VDFS), and run by the Cambridge Astronomy Survey Unit (CASU) ${ }^{2}$. The pipeline delivers science-ready stacked images and tiles, as well as photometrically and astrometrically calibrated source catalogs (Irwin et al. 2004). A total of 3.2 million sources were detected in the VISTA Orion Survey, and $\sim 155000$ in tile No. 12 used for this work. The catalog also provides, for each source in each filter, a morphological parameter (FLAG) equal to -1 for point-like sources, 1 for extended sources, -2 for borderline point-like sources, and $-7,-9$ for problematic detections, e.g., sources partly saturated or whose magnitude is contaminated by bad pixels inside the aperture used for the photometry extraction, or truncated because the source is very close to the mosaic borders. The astrometric accuracy in the source catalog is $\sim 0.2$ with respect to the UCAC4 catalog (Zacharias et al. 2013). Stellar sources typically show a FWHM of 0.' 6-0.' 8 . The instrumental magnitudes are obtained through aperture photometry and calibrated onto the VISTA system via nonsaturated 2MASS stars in the field. Absolute photometric uncertainties are below $5 \%$ and the $5 \sigma$ limiting magnitudes, completeness, and saturation limits in each filter are listed in Table 1 for the specific case of tile No. 12 . The achieved magnitude limits in $J H K_{\mathrm{S}}$ are $\sim 3$ mag deeper than 2MASS. The approximate completeness limit in each filter was derived as the point where the histogram of the magnitudes (Fig. 3) diverges from the dotted line, which represents the linear fit to the logarithmic number of objects per magnitude bin, calculated over the intervals of good photometric accuracy (Santiago et al. 1996; Wainscoat et al. 1992). To estimate stellar mass limits from our saturation and completeness limits, we compare our limits with the theoretical isochrones by Baraffe et al. (1998) and Chabrier et al. (2000). Since the isochrones are provided for the Johnson-Cousins photometric system, which is different from the VISTA photometric system, we converted them to the VISTA photometric system as

\footnotetext{
2 http://apm49.ast.cam.ac.uk/surveys-projects/vista/ vdfs
} 


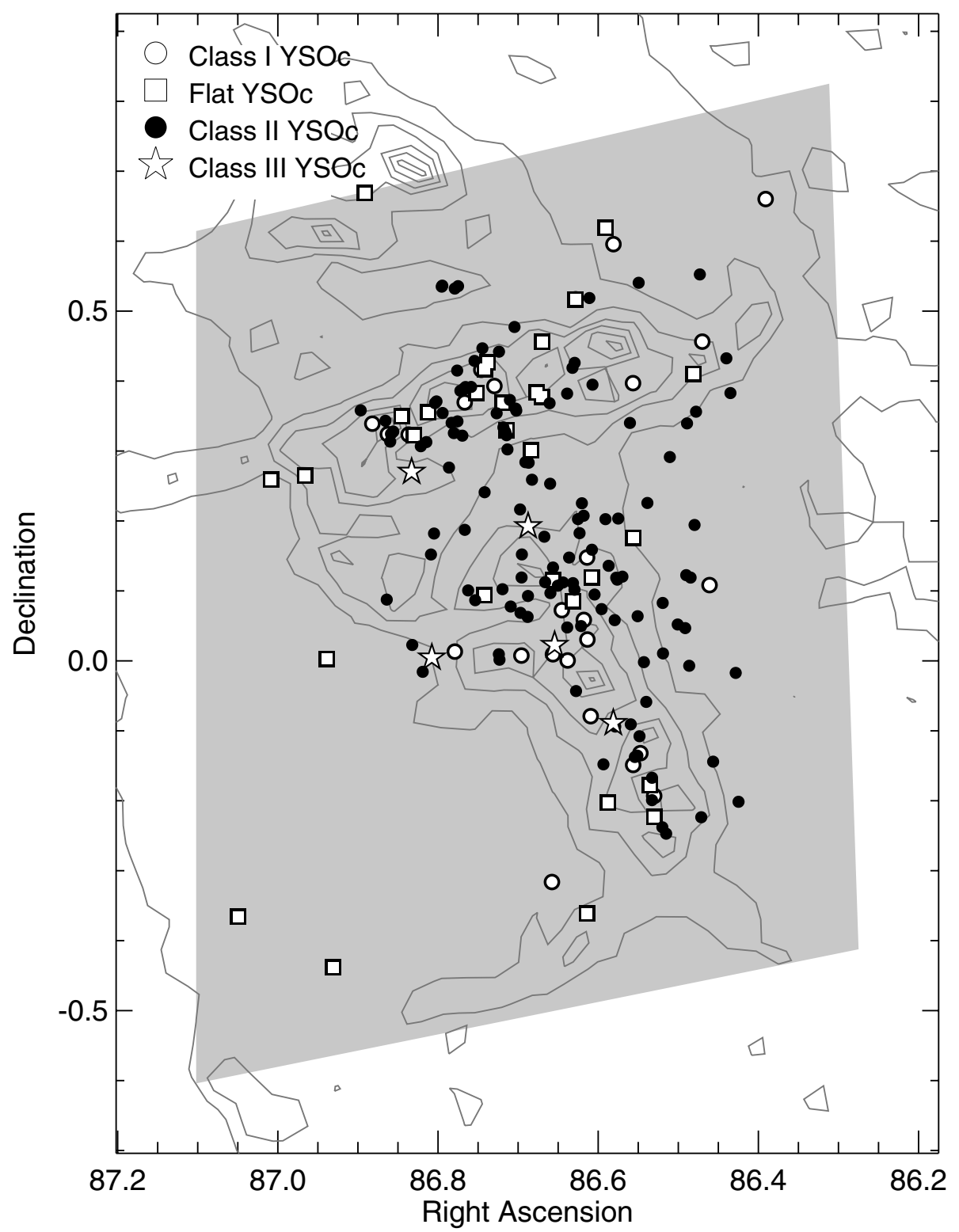

Fig. 1. Spatial distribution of the YSO candidates as a function of Lada classes over-plotted on the contours from the VISTA extinction map (solid lines). The contour levels of extinction $\left(A_{K}\right)$ are from 0.01 to $2 \mathrm{mag}$, in steps of $0.2 \mathrm{mag}$. The shaded area displays the regions observed with VISTA (tile No. 12).

Table 1. Filter central wavelength, saturation limit, limiting magnitude at the $5 \sigma$ level and completeness limit for the photometry of sources in tile No. $12(\mathrm{~L} 1630 \mathrm{~N})$.

\begin{tabular}{ccccc}
\hline \hline Filter & $\begin{array}{c}\lambda_{\mathrm{C}} \\
(\mu \mathrm{m})\end{array}$ & $\begin{array}{c}\text { Saturation } \\
\text { limit }\end{array}$ & $\mathrm{Mag}_{5 \sigma}$ & $\begin{array}{c}\text { Completeness } \\
\text { limit }\end{array}$ \\
\hline$Z$ & 0.877 & 13.5 & 22.5 & 22.3 \\
$Y$ & 1.020 & 12.0 & 21.1 & 21.5 \\
$J$ & 1.252 & 11.0 & 20.3 & 20.5 \\
$H$ & 1.645 & 11.0 & 19.3 & 19.5 \\
$K_{\mathrm{S}}$ & 2.147 & 10.0 & 18.5 & 18.5 \\
\hline
\end{tabular}

described in Appendix A. We estimate that our survey should have detected, for a population as young as $\sim 2 \mathrm{Myr}$ at a distance of about 400 pc (i.e., the case of L1630 N) essentially all objects from $\sim 1 M_{\odot}$ down to $\sim 5$ Jupiter masses $\left(\sim 0.0045 M_{\odot}\right)$ in a region showing less than 1 mag of visual interstellar extinction.

\subsection{Spitzer data}

The Orion clouds were observed by the Spitzer Space Telescope (Fazio et al. 2004; Rieke et al. 2004) as part of the guaranteed time observation (GTO) programs PID 43, 47, 50, 58, 30641 , and 50070 . The extraction of point source photometry from this survey in the four IRAC bands and the MIPS $24 \mu \mathrm{m}$ band and an overview of the basic properties of the resulting point source catalog have been presented by Megeath et al. (2012). The cata$\log$ contains 298405 sources that are detected in at least one of the IRAC bands or in the MIPS $24 \mu \mathrm{m}$ band, the limiting magnitudes at the $10 \sigma$ level are roughly 16.5, 16.0, 14.0, 13.0 and 8.5 at 3.6, 4.5, 5.8, 8 and $24 \mu \mathrm{m}$, respectively (see Fig. 2 by Megeath et al. 2012). The catalog spatially exhibits varying completeness due to confusion with nebulosity and crowding of point sources in dense clusters (see Figs. 3, 4 by Megeath et al. 2012). The Orion IRAC/MIPS maps are broken into several fields centered on the regions of strong ${ }^{13} \mathrm{CO}$ emission (Miesch \& Bally 1994). 


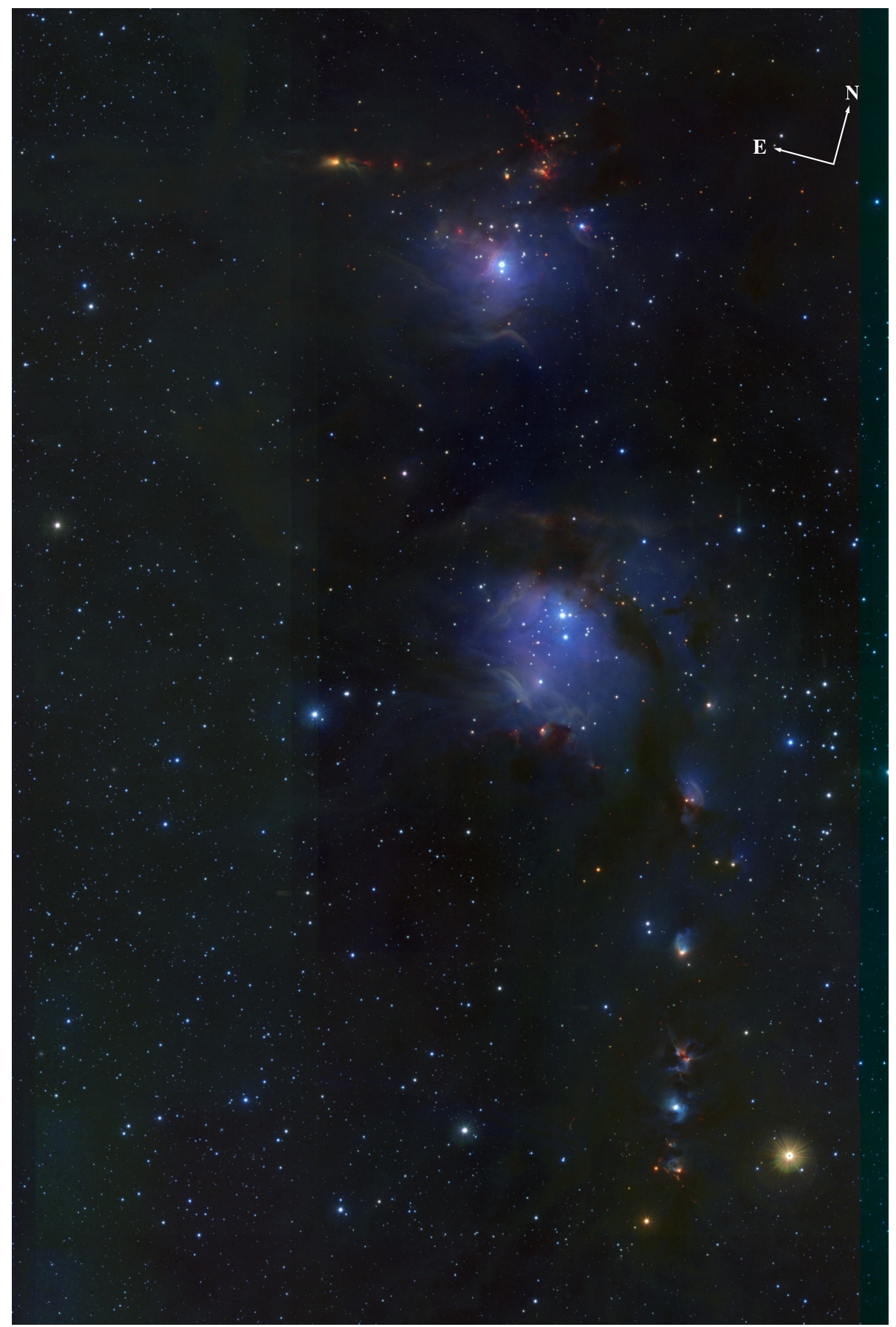

Fig. 2. VISTA three color (ZJKs) mosaic image of L1630 $\mathrm{N}$ on a logarithmic display. The image covers the gray shaded area of Fig. 1.

We used a subset of the general catalog, covering an area of $2.58 \mathrm{deg}^{2}$ around L1630 N.

\section{Selection of YSO candidates}

The $J-H$ vs. $H-K_{\mathrm{S}}$ color-color (CC) diagram is traditionally used to select low-mass YSO candidates (YSOc) on the basis of near-IR data, because young K/M type stars exhibit a narrow range of colors in this diagram and, in particular, an $H-K_{\mathrm{S}}$ excess mainly arising from their circumstellar disk and/or infalling envelope (Meyer et al. 1997; Luhman \& Rieke 1999; Lee et al. 2005). However, near-IR colors of YSOs may be mimicked, to some extent, by highly reddened stellar photospheres of older main-sequence dwarf stars in the field and, hence, the selected sample might be contaminated. On the other hand, the identification of YSOs on the basis of mid- to far-IR colors alone is not trivial because the YSO colors in this wavelength regime are very similar to those of many background galaxies (Sect. 3.1 by Evans et al. 2009). Thus, to select YSOs in L1630 N we adopt the approach by Harvey et al. (2007a), based on the combined use of $\mathrm{JHK}_{\mathrm{S}}$ photometry and Spitzer IRAC/MIPS colors. Using Spitzer observations of the Serpens star-forming region and the SWIRE catalog of extragalactic sources (Lonsdale et al. 2003), these authors defined the boundaries of the disk-bearing YSO locus in several IRAC/MIPS color-magnitude (CM) and CC diagrams. Their criteria have proven to provide an optimal separation between disk-bearing YSOs (mainly Class II, Class I and flat-spectrum objects), reddened field stars, and galaxies, with 

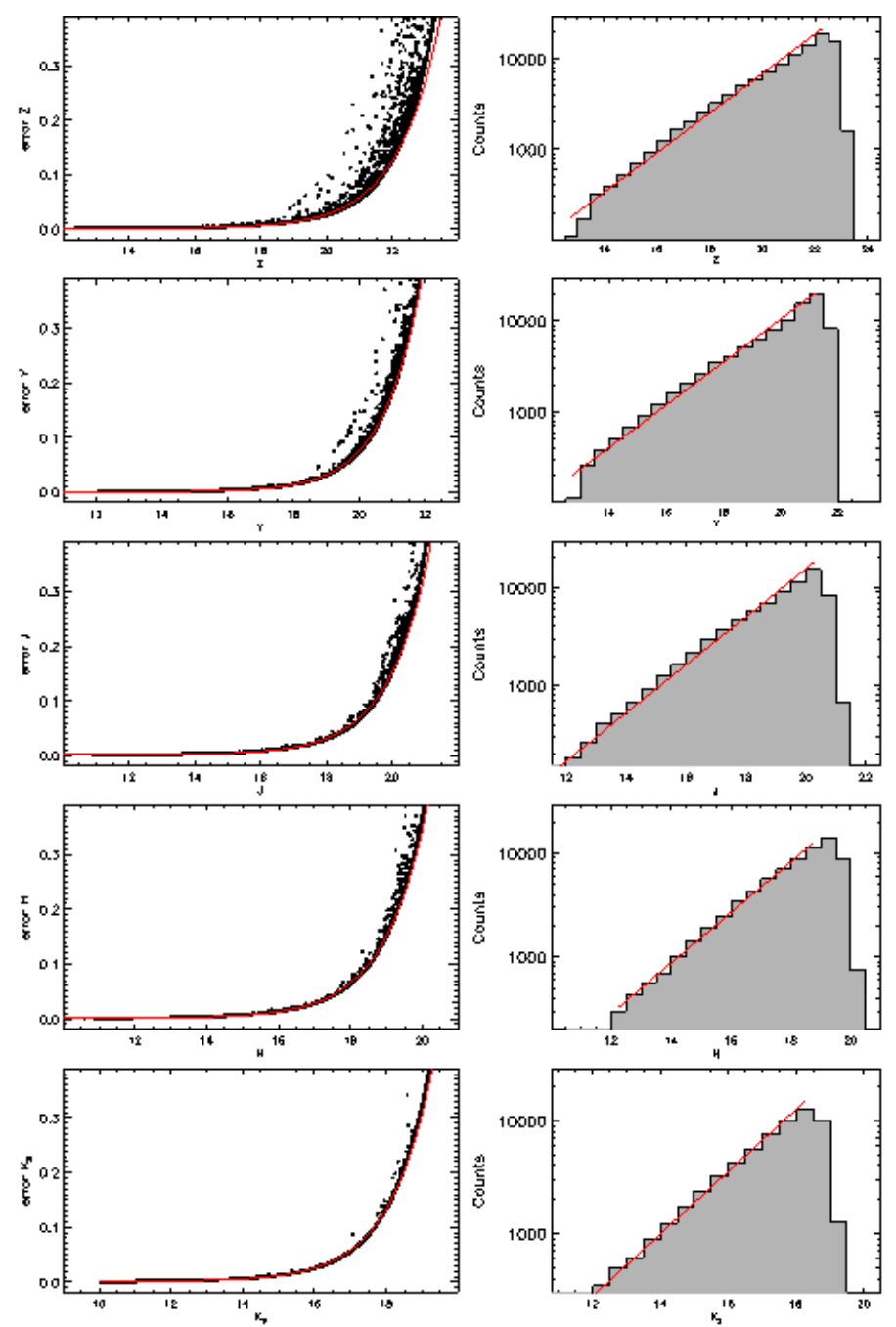

Fig. 3. Left panels: photometric errors as a function of magnitudes and relative exponential fit (continuous line) for nonsaturated sources detected by the VISTA Orion Survey tile No. 12 in the ZYJHK filters. Right panels: number of detection as a function of magnitude. The line shows the linear fit to the logarithmic number distribution of magnitudes, which is used to find the turning point of the distribution, indicating our completeness limit.

the fraction of remaining contaminants (mainly background field dMe dwarfs, a few K/M-type giants, and Be and AGB stars) estimated to be around 30\% (e.g., Spezzi et al. 2008; Oliveira et al. 2009; Cieza et al. 2010). These criteria have been applied to select YSO candidates in all star-forming regions observed within the frame of the Spitzer c2d (Evans et al. 2009) and Spitzer Gould Belt ${ }^{3}$ Legacy surveys (e.g., Spezzi et al. 2011; Hatchell et al. 2012; Dunham et al. 2013).

We first matched our VISTA catalog for tile No. 12 with the Spitzer catalog by Megeath et al. (2012) using a matching radius of $3^{\prime \prime}$, larger than the astrometric accuracy of our mosaics (Sect. 2.1) and corresponding to twice the typical FWHM of sources in IRAC maps ${ }^{4}$. Then, we applied the YSO selection method by Harvey et al. (2007a) to the matched catalog, containing $\sim 58500$ sources with complete $Z Y J H K_{\mathrm{S}}$, IRAC 3.6, 4.5, 5.8 and $8 \mu \mathrm{m}$ and MIPS-24 $\mu \mathrm{m}$ photometry. A detailed

\footnotetext{
3 http://www.cfa.harvard.edu/gouldbelt

4 IRAC Instrument Handbook. See http://irsa.ipac.caltech. edu/data/SPITZER/docs/irac/iracinstrumenthandbook/
}

review of the selection method can be found in Harvey et al. (2007a,b). Briefly, the selection method consists in the definition of an empirical probability function, which depends on the relative position of a given source in several CC and CM diagrams, where diffuse boundaries have been determined to obtain an optimal separation between YSOs and galaxies. Figure 4 (left panels) shows the VISTA/Spitzer CC and CM diagrams used to select the YSO candidates in L1630 N. Note that the method requires detection in all IRAC bands and in MIPS-24 $\mu \mathrm{m}$ with a $\mathrm{S} / \mathrm{N}$ higher than 3 to classify an object as a YSO candidate or a background galaxy. Diskless YSOs, i.e., Class III sources, are usually rejected by the selection method. Moreover, older field objects with no IR excess emission are rejected a priori because their IR colors are comparable with normal photospheric colors, e.g., $K_{\mathrm{S}}-[4.5]<-0.1$, [8]-[24] $<0.1$, [4.5]-[8] $<0.2$ (Harvey et al. 2007b). In addition to the criteria by Harvey et al. (2007a), we used the VISTA morphological parameter (FLAG; Sect. 2.1) to distinguish between point-like (FLAG $=-1)$ and extended $(\mathrm{FLAG}=1)$ YSO candidates; YSO candidates truncated and/or contaminated in VISTA passbands $(-9 \leq$ FLAG $\leq-2)$ could not be classified. Although clearly extended sources in the VISTA mosaic are more likely to be galaxies, we can not exclude them a priori, because YSOs still surrounded by significant circumstellar material might appear fuzzy/extended at IR wavelengths. Thus, we establish the YSO or galaxy nature on the basis of the above mentioned probability function alone, which depends exclusively on the VISTA/Spitzer colors of the sources.

We find 188 YSOc in L1630N, shown in Fig. 4 (left panels) as red dots, squares, and asterisks for point-like, extended, and morphologically unclassified sources, respectively.

\subsection{On the contamination of the YSO candidates sample}

We adopted a statistical method to distinguish YSOs from extragalactic contaminants and field stars, hence, our candidates sample could be still slightly contaminated.

The number of interloping stars can be probed using analytic models of the Galactic stellar distribution, i.e., simulations of the expected properties of stars seen toward a given direction of the Galaxy over a given solid angle. We performed this exercise using the Galaxy model by Robin et al. (2003) and their online tool $^{5}$. In the temperature range of our candidates, foreground stars are expected to be main-sequence cool dwarfs, whereas red giants are expected to dominate the background population. Assuming a cluster distance of $400 \mathrm{pc}$ and a typical extinction of $A_{\mathrm{V}} \approx 1 \mathrm{mag}$ due to the $\mathrm{L} 1630 \mathrm{~N}$ cloud itself, we expect some 50 foreground dwarfs in the $1 \times 1.5 \mathrm{sq}$. deg area observed by VISTA (tile n. 12) with apparent $K_{\mathrm{S}}$ magnitude between 7 and 18.5 and spectral types of M0 to M9, i.e., the magnitude and spectral range corresponding to members of L1630 N detectable by our survey $\left(\lesssim 1 M_{\odot}\right.$; Sect. 2.1$)$. Only a handful $(\sim 3)$ of background giants are expected to be found in the locus occupied by the cluster members because they generally appear much brighter than L1630 N members in the same effective temperature range. We thus conclude that only foreground cool mainsequence stars can contribute noticeably to the contamination of our candidate sample, but the contamination level is at most 25$30 \%$. We note that contamination is higher $(\sim 50 \%)$ in the substellar regime $\left(\sim 0.1 M_{\odot}\right.$, i.e., $\left.K_{\mathrm{S}} \approx 13 \mathrm{mag}\right)$, and lower in the stellar regime $(\sim 20 \%)$. On the other hand, as seen in Fig. 1, the YSOc very clearly follow the cloud extinction contours, which is not expected for a randomly distributed foreground population.

\footnotetext{
5 http://model.obs-besancon.fr/
} 
A\&A 581, A140 (2015)
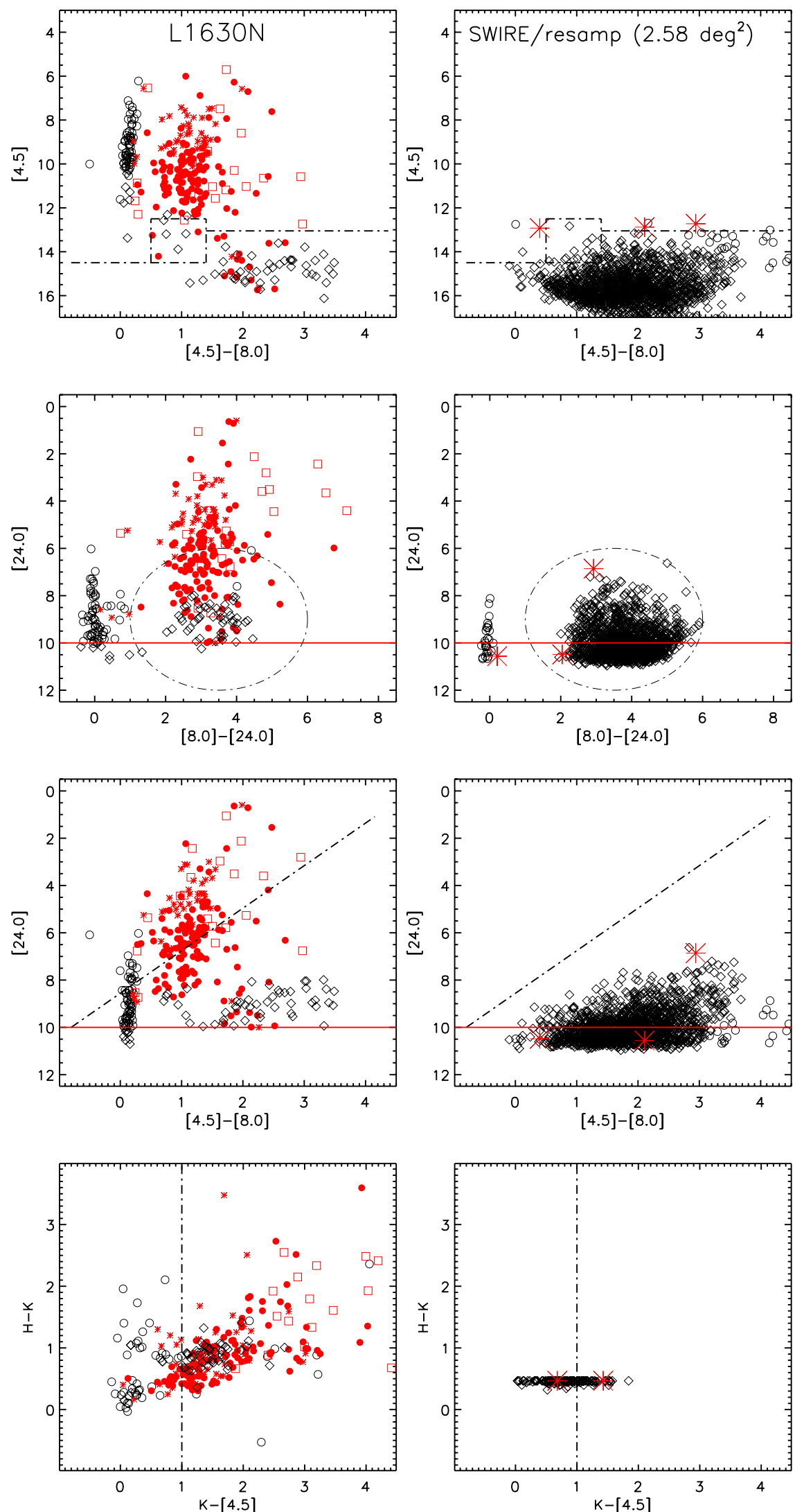

Fig. 4. Left panels: VISTA/Spitzer CM and CC diagrams for L1630 N. The dot-dashed lines show fuzzy limits with exponential cutoffs that define the YSO candidate selection criterion in the each diagram, excluding contamination from galaxy (diamonds) and field stars presenting normal photospheric colors (circles). The continuous lines show hard limits, objects fainter than which are excluded from the YSO category. Point-like and extended YSO candidates are indicated by dots and squares, respectively; YSO candidates with no VISTA morphological classification are indicated by asterisks. Right panels: 2MASS/Spitzer CM and CC diagrams for the SWIRE catalog (Sect. 3.1). Three objects from the SWIRE catalog (marked as astericks) are classified as YSO candidates, according to our selection criteria. 
Also, we find almost all of our YSOc consistent with infrared excess sources of Class II or earlier (see Sect. 5). Therefore, we conclude that the true contamination of our sample is very low.

For a statistical estimate of possible remaining extragalactic contaminants, we used the Spitzer Wide-area Infrared Extragalactic (SWIRE Surace et al. 2004) catalog from the observations of the ELAIS N1 field (Rowan-Robinson et al. 2004). The SWIRE catalog was trimmed and resampled as accurately as possible to match the spatial extent $\left(2.58 \mathrm{deg}^{2}\right)$ and sensitivity limits (Sect. 2.2) of the Spitzer observations in L1630 N. Moreover, the photometry of sources in the SWIRE catalog was edited to simulate the interstellar extinction in the direction of $\mathrm{L} 1630 \mathrm{~N}$, as expected on the basis of the VISTA extinction map (Sect. 7.1). The $J H K_{\mathrm{S}}$ for the SWIRE sources are recovered from the 2MASS catalog (Skrutskie et al. 2006). Evans (2008) and Harvey et al. (2007a) give further details on how the trimmed resampled SWIRE comparison catalog was created. The selection criteria applied to the comparison resampled SWIRE data lead to the conclusion that only three (i.e., less than $2 \%$ ) of the selected YSO candidates in L1630 N may be background galaxies (Fig. 4). This result is similar to what Harvey et al. (2007a) and Alcalá et al. (2008) found for the Serpens and Cha II molecular clouds, respectively. Indeed, all the 188 candidates have been visually inspected in the VISTA images and we find that only two of them are clearly galaxies; these two candidates have been neglected in the subsequent analysis. All the remaining 186 candidates appear point-like or almost point-like in all our images and their photometry is not contaminated by nearby saturated stars, or any other artifact that might affect our selection criterion. A few of them $(\sim 3 \%)$ present a close companion in the VISTA images not resolved in the Spitzer images and, hence, their Spitzer fluxes might be contaminated. We can not discharge these candidates a priori because one or both objects in the system might still be young and, hence, responsible for the observed IR excess emission.

This leads us to a remaining caveat in our selection method. Our YSO candidates might be members of binary/multiple systems too close to be resolved with VISTA/Spitzer and, hence, affect the measured photometry. As seen in Sect. 2.1, our candidates are expected to have masses $\lesssim 1 M_{\odot}$. The multiplicity fraction for stars in this mass regime is estimated to be between $20 \%$ and $40 \%$, depending on the actual mass of the primary star and the separation range (Duquennoy \& Mayor 1991; Mason et al. 1998; Basri \& Reiners 2006; Lada 2006). However, higher resolution imaging or spectroscopy would be needed to assess the actual multiplicity fraction in L1630 N.

\subsection{Comparison with previous surveys}

Before our study, a census of the young stellar population in L1630 N was presented by Flaherty \& Muzerolle (2008) and Fang et al. (2009). Flaherty \& Muzerolle (2008) identified 69 cluster members with a rather spread spatial distribution, i.e., not confined to regions of dense gas and dust. For 67 of these members, they derived accurate spectral type and luminosity and estimated a median age of $2 \mathrm{Myr}$, and a large fraction of stars with infrared excess actively accreting (79\%). Using a mix of criteria (presence of $\mathrm{H} \alpha$ emission, Li I absorption, or IR excess), Fang et al. (2009) selected and analyzed 132 PMS stars in the general direction of the clusters. This list includes practically all the PMS stars studied by Flaherty \& Muzerolle (2008). For 111 stars, Fang et al. (2009) provide a classification in terms of their IR-excess as "thick disk, transitional disk, thin disk, and no disk". Most of the 21 objects missing IR classification lack any information on Li I absorption and were selected as PMS stars by Fang et al. (2009) only because $\mathrm{H} \alpha$ is detected in emission, although rather weak considering their spectral types (White \& Basri 2003).

Our YSOc sample consists of 186 objects. In Table A.2 we report their coordinates and VISTA + Spitzer photometry. The VISTA/Spitzer selection criteria recovered 50 out of the 69 (i.e., $\sim 75 \%$ ) PMS stars listed by Flaherty \& Muzerolle (2008), specifically 10 weak T Tauri stars (WTTs) and 40 classical T Tauri stars (CTTs). Likewise, our criteria recover 82 of the 132 objects in Fang et al. (2009; i.e., $62 \%$ of the whole sample, but $74 \%$ of the sample with IR classification). Most of the recovered objects by the VISTA/Spitzer selection criteria in both catalogs can be classified as Class II YSOs. Our survey missed about 50 of the previously known YSOs in the area, i.e., $~ 21 \%$ of the YSO population $(50$ of $186+50)$, the vast majority of which are WTTs or objects without disks, i.e., Class III YSOs. In Table A.2c we mark the YSOc already identified by Flaherty \& Muzerolle (2008) and/or Fang et al. (2009).

Although the selection criteria and color cuts presented here are different from those by Megeath et al. (2012) it is also interesting to compare our results with their selection, since we gathered the Spitzer photometry from their catalog (see Sect. 2.2). In our studied area there are 257 sources selected by Megeath et al. (2012) as possible YSOs, but 75 of these lack information in at least one IRAC band or at $24 \mu \mathrm{m}$. Since our selection criteria require the detection in all IRAC bands and at $24 \mu \mathrm{m}$, we can classify 182 of the Megeath et al. (2012) sources in the region. With our methods we thus recover 162 YSOs, meaning that our criteria miss 20 of the Megeath et al. (2012) sources. Most of them are classified as possible protostar candidates by Megeath et al. (2012) and are distributed in regions of high sellar density. Thus, our criteria recover about $90 \%$ of the Megeath et al. (2012) sources.

\section{Luminosity function and characteristic stellar mass}

The stellar luminosity can be used as a poor but still useful firstorder proxy for mass, assuming that most of the stars formed more or less at the same time. Before using the range of luminosities to provide an estimate of the mass range, we determined the degree of completeness of the YSO candidate sample in L1630 N. Our selection criteria ultimately rely on the VISTA and Spitzer IRAC 3.6-8 $\mu \mathrm{m} / \mathrm{MIPS} 24 \mu \mathrm{m}$ detection of the objects and on the quality of this detection. Thus, to investigate the expected number of low-luminosity objects and infer the typical mass distribution of our YSOc sample, we have to take the completeness of these two data sets into account.

\subsection{The 1-30 $\mu \mathrm{m}$ bolometric luminosity function}

To estimate the completeness of the Spitzer observations in the L1630 N, we used the same approach as Harvey et al. (2007a), which has been applied to all c2d/GB clouds (e.g., Alcalá et al. 2008; Merín et al. 2008; Spezzi et al. 2011).

We first derive the total infrared luminosity of our YSOc through integration over their SED flux between 1 to $30 \mu \mathrm{m}$; the total flux was converted to luminosity assuming a distance of 400 pc. We then applied the Harvey et al. (2007a) completeness correction factors for the c2d survey to our YSO candidate samples. Harvey et al. (2007a) estimated the completeness of the $\mathrm{c} 2 \mathrm{~d}$ catalogs by comparing, for each luminosity bin, the number 


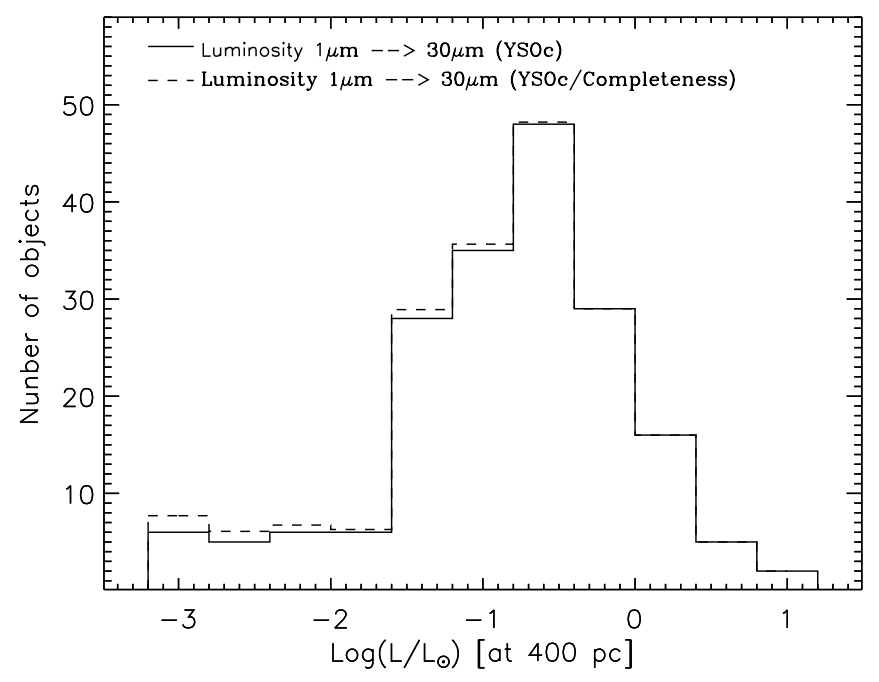

Fig. 5. Luminosity distribution for the YSOc in L1630 N (solid histogram). The plotted luminosities were determined as in Harvey et al. (2007a), i.e., by integration of the SEDs from 1 to $30 \mu \mathrm{m}$. The corrected luminosity distribution, determined by applying completeness factors at each luminosity bin as in Harvey et al. (2007a), is over-plotted (dashed histogram).

of counts from a trimmed version of the deeper SWIRE catalog of extragalactic sources (assumed to represent $100 \%$ completeness by c2d standards) with the number of counts for the $\mathrm{c} 2 \mathrm{~d}$ catalogs in Serpens. This completeness correction can be applied to our Spitzer catalog of L1630 N because its photometric depth (Sect. 2.2) is similar to the one of the c2d catalogs (in both cases the $10 \sigma$ limit is $\sim 16.5 \mathrm{mag}$ for IRAC $3.6 \mu \mathrm{m}$ and $\sim 8.5 \mathrm{mag}$ for MIPS $24 \mu \mathrm{m}$; compare Figs. 23 and 26 by Evans 2008; with Fig. 2 by Megeath et al. 2012). Figure 5 shows the $1-30 \mu \mathrm{m}$ bolometric luminosity function (BLF) for YSOc in L1630 N before (solid line) and after (dashed line) correction for completeness, and suggests that we are missing only a few $(<5)$ of additional low-luminosity sources with $\log \left(L / L_{\odot}\right)<-1.7$. These objects have been missed by our selection either because they are below the noise level of the Spitzer observations or because they are located within the galaxy loci of the CM diagrams (Fig. 4). In conclusion, our YSO candidate samples in L $1630 \mathrm{~N}$ is fairly complete. The luminosity histogram suggests a completeness better than $\sim 95 \%$ at luminosities down to $0.01 L_{\odot}$, which corresponds to a mass of $0.02 M_{\odot}$ for 2 Myr old stars, according to the PMS evolutionary tracks by Baraffe et al. (1998) and Chabrier et al. (2000). The peak of the luminosity function appears at $0.25 L_{\odot}$, which corresponds to a $0.4 M_{\odot}$ star (i.e., spectral type M3 at an age of $2 \mathrm{Myr}$ ). A very peculiar characteristic of the L1630 N 1-30 $\mu \mathrm{m}$ bolometric luminosity function is that it shows a significant number $(\sim 35 \%)$ of low- and very low-luminosity objects $\left(\log \left(L / L_{\odot}\right) \lesssim-1\right)$. A similar tail of low-luminosity objects was noted in the Lupus I, III, and IV star-forming regions ( 40\% Merín et al. 2008; Comerón 2008), while it is not observed in other c2d/GB clouds, such as Cha II ( $\sim 15 \%$ Alcalá et al. 2008), Lupus V and VI (10-20\% Spezzi et al. 2011) and Serpens (Harvey et al. 2007a).

\subsection{K-band luminosity function}

To further investigate/confirm the stellar mass distribution of the L1630 N YSO population, we also constructed its $K$-band luminosity function (KLF). We choose the KLF rather than the $J$ or $H$-band luminosity functions to minimize the effects of

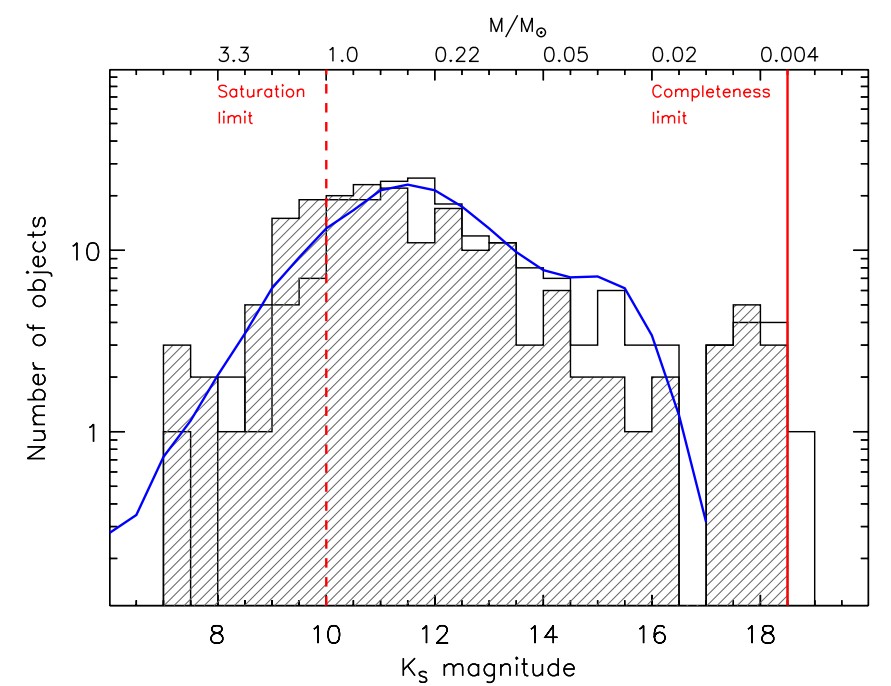

Fig. 6. The L $1630 \mathrm{~N} K$-band luminosity function (KLF) before (empty histogram) and after correction for interstellar extinction (line-filled histogram). The labels in the top $x$-axis indicate the corresponding stellar mass according to the VISTA 2 Myr isochrone (Appendix A). The continuous and dashed vertical lines indicate the completeness and the saturation limit of the VISTA $K$-band photometry, respectively. The continuous curve is the KLF of the Trapezium cluster (Muench et al. 2002), scaled to the peak of the L1630 N KLF.

extinction, to maximize our sensitivity to intrinsically red, lowluminosity members of this cluster, and to make detailed comparisons to the KLF of the nearby Trapezium cluster presented by Muench et al. (2002). We did not correct the $K$-band fluxes for excess emission, but this should not affect the comparison with the Trapezium Cluster KLF, because Muench et al. (2002) do not correct for disk excess flux neither. The majority of stars in the Trapezium sample are disk-sources, and the $H-K$ color distribution of the whole Trapezium sample is very similar to the $H-K$ color distribution of our YSO sample in L1630, indicating a similar disk excess nature for the samples.

In Fig. 6 we present the observed and dereddened KLF of L1630 N. We use relatively wide bins $(0.5 \mathrm{mag})$ that are much larger than the photometric errors (Fig. 3), and, for each YSOc, adopt the visual extinction derived from the VISTA extinction map (Sect. 7.1). The $K$-band excess could add $\sim 0.5 \mathrm{mag}$, on average, to the observed $K$-band magnitude (Meyer et al. 1997), i.e., about the same size as the KLF binsize. However, this does not have any significant affect on our conclusions on the KLF shape, as the excess is a property over the entire luminosity range (i.e., there is no singular effect on an individual mass regime of the KLF), and the steady decline in the substellar regime discussed below is a robust result. We also indicate in Fig. 6 the $K$-band saturation limit and limiting magnitude of our VISTA catalog (Table 1) and overplot, for comparison purposes, the Trapezium KLF as derived by Muench et al. (2002), arbitrarily scaled to the peak of the L1630 N KLF.

The KLF of L1630N shows a broad peak between 10.5 and $12 \mathrm{mag}$, i.e., $0.3-0.7 M_{\odot}$ at the cluster distance and age according to the 2 Myr isochrone by Baraffe et al. (1998) and Chabrier et al. (2000) converted to the VISTA photometric system (Appendix A). Then, it steadily declines to the Hydrogenburning limit $\left(\sim 0.1 M_{\odot}\right.$, i.e., $\left.K_{\mathrm{S}} \approx 13 \mathrm{mag}\right)$. Below this limit, we count a fraction of $28 \%$ substellar YSOs 6 . However, we note that

6 This fraction is computed as the number of substellar objects over the total number of YSOc. 
the expected contamination from field stars mimicking the colors of BDs is expected to be $\sim 50 \%$ (Sect. 3.1), while it is lower in the stellar regime $(\sim 20 \%)$ and, hence, the actual fraction of substellar objects in L1630 N could be as low as $20 \%$. Thus, the KLF of L1630 N indicate a stellar mass distribution consistent with the 1-30 $\mu \mathrm{m}$ BLF. Moreover, it appears remarkably similar to the Trapezium KLF (see Fig. 11a by Muench et al. 2002), which presents a broad peak around $0.6 M_{\odot}$ and then declines into the substellar regime, with the fraction of substellar objects at $\sim 22 \%$. Muench et al. (2002) also reported a significant secondary peak around 10-20 Jupiter masses $\left(\sim 0.02 M_{\odot}\right)$. Although we do observe a similar fraction of substellar objects, the presence of this secondary peak is not obvious in the KLF of L1630 N, which appears to keep its steady decline down to our completeness limit $\left(\sim 0.0045 M_{\odot}\right.$, i.e., $\left.K_{\mathrm{S}} \approx 18.5 \mathrm{mag}\right)$.

The mass function shape of the Trapezium cluster in the substellar regime has long been debated because the large fraction of brown dwarfs (BDs) with respect to other nearby starforming regions ( 15\%; Briceño et al. 2002; López Martí et al. 2004; Spezzi et al. 2007, 2008, 2009) could be an affect of spatial and photometric incompleteness of the surveys conducted so far. However, more and more complete surveys are now available and, still, there is no universal agreement on the behavior of the mass function over the substellar regime. For a complete collection of BD fraction measurements in nearby starforming regions and a discussion on possible trends, see Scholz et al. (2012) and references therein. These authors compare the star-to-BD ratios $\left(R_{\mathrm{star} / \mathrm{BD}}\right)$ for various star-forming regions (see their Table 5). It appears more and more evident that, on one hand, there are clusters like IC 348, T association like Taurus, Chamaeleon, $\rho$-Ophiuchus, etc., with a low number of substellar objects $\left(R_{\text {star/BD }}\right.$ in the range 5 to 8$)$ and, on the other hand, there are more massive clusters, such as Trapezium, the ONC, NGC 1333, Upper Scorpius, etc., in which this number is much higher $\left(R_{\mathrm{star} / \mathrm{BD}} \approx 2-4\right)$. L1630 N, like other clusters in the Orion complex (Trapezium and the ONC), would belong to this last category, with $R_{\text {star/BD }} \approx 2.5-3.9$, depending on the actual contamination level. The variation of the fraction of substellar objects observed from region to region possibly indicates environmental effects on their formation mechanism, a key point of the current star formation theory still under debate (e.g., Whitworth et al. 2007).

\section{Lada classes, disk fraction, and disk lifetimes}

In this section we revise the disk properties of the young stellar population in NGC 2068/2071 on the basis of our YSO candidates sample, and in comparison with the results by Flaherty \& Muzerolle (2008).

To investigate the disk properties of our YSO candidate sample, we adopted the Lada classification (Lada \& Wilking 1984) based on the SED slope $(\alpha)$ of the line joining the flux measurements at $2.2 \mu \mathrm{m}$ ( $K$-band) and MIPS-24 $\mu \mathrm{m}$. In particular, we used the Lada's class separation as extended by Greene et al. (1994), i.e., $\alpha \geq 0.3$ for Class I, $-0.3 \leq \alpha<0.3$, for flatspectrum sources, $-1.6 \leq \alpha<-0.3$, for Class II sources, and $\alpha<-1.6$, for Class III sources. We report in Table A. 2 the Lada class computed for each YSOc and give in Table 2 the statistics for the entire YSOc sample in L1630 N. As shown in Fig. 7, the dominant objects in L1630 N are those of Class II $(68 \%)$, followed by flat-spectrum (16\%) and Class I (13\%) sources, with only a minority being Class III sources (3\%). The distribution of YSOs over class supports the young age estimated for this star-forming region. The ratio of the number of Class I and
Table 2. Summary of Lada classes in L $1630 \mathrm{~N}$ and estimated lifetime for each phase.

\begin{tabular}{ccc}
\hline \hline Lada class & No. of YSO candidates & $\begin{array}{c}\text { Lifetime } \\
(\mathrm{Myr})\end{array}$ \\
\hline I & $25(13 \%)$ & 0.40 \\
Flat & $30(16 \%)$ & 0.48 \\
II & $126(68 \%)$ & $2^{\dagger}$ \\
III & $5(3 \%)$ & - \\
\hline
\end{tabular}

Notes. ${ }^{(\dagger)}$ Assumed lifetime for the Class II phase (Evans et al. 2009).

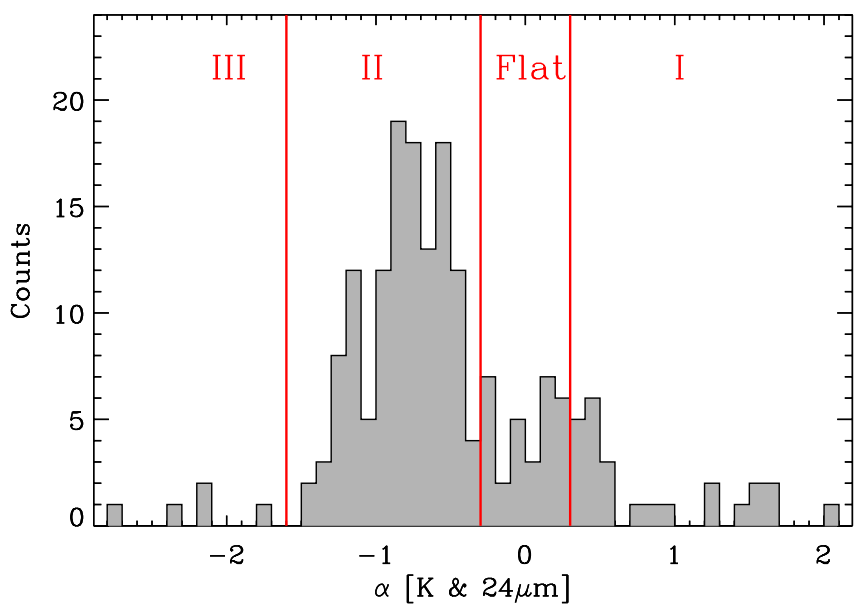

Fig. 7. $\alpha$-slope distribution of YSOc in L1630 N. The vertical lines indicate the intervals defining the four Lada classes. The population is largely dominated by Class II objects.

flat-spectrum sources to the number of Class II and Class III sources is 0.42, similar to the ratio measured in Serpens (Harvey et al. 2007a) and other clouds of similar age surveyed by the Spitzer c2d survey (Evans et al. 2009). The total observed fraction of objects with thick disks and/or envelopes (Class I to II) for our sample is on the order of $97 \%$, while those with thin or no disk (Class III) represents only $\sim 3 \%$ of the sample. The total disk fraction is considerably higher than the values derived in other regions of similar age (e.g., in IC 348; Lada 2006) and this would make $\mathrm{L} 1630 \mathrm{~N}$ a clear outlier with respect to the typical disk fraction vs. age trend (e.g., see Figs. 1 and 4 by Haisch et al. 2001; Fedele et al. 2010, respectively).

Contamination from field stars, which could be as high as 25-30\% (Sect. 3.1), should not heavily affect the relative number of objects with and without a disk because there is no reason to assume that this kind of contamination affects one Lada class more than the others. Background galaxies preferentially mimic the colors of YSOs with thick disks/envelope, but they may account for $2 \%$ of our YSOc sample at most and cannot justify the high number of Class I to II objects.

However, one may wonder whether our census might have missed a significant number of diskless YSOs. This would be possible because the $\mathrm{c} 2 \mathrm{~d}$ criteria select only IR excess objects. A direct way to investigate the number of diskless YSOs missed in our survey is to compare our results with those of deep X-ray observations, which are the most secure way to trace the population of Class III objects. By merging many Chandra pointed observations in a region centered between NGC 2068 and HH2426, Principe et al. (2014) obtained a very deep X-ray image with an equivalent exposure time of about $240 \times 10^{3} \mathrm{~s}$. In an area of about 0.12 sq. deg, they detected 52 X-ray sources, 32 of which can be identified with Class III or transition objects. Six of these 
objects have been recovered in the same area by our selection, but five were classified as Class III and one as Class II by our work. Considering that transition objects may mimic colors of Class III objects, the conclusion is that our selection misses the actual number of diskless YSOs by a factor of about 5 or 6 . This is in perfect agreement with the estimated number of missed diskless YSOs in the c2d surveys (see Sect. 3.2 in Evans et al. 2009). Hence, we are most likely missing 25-30 Class III YSOs (i.e. $20 \%$ ) in our sample (see Table 2), which is also consistent with the fraction of diskless YSOs missed by our criteria in the Flaherty \& Muzerolle (2008) and Fang et al. (2009) samples (see Sect. 3.2). Certainly, to obtain a real full census of Class III objects in the area, a much larger scale deep X-ray survey would be needed. Since these kinds of observations are currently not available, we use the above correction as a first order estimate, which leads to an expected fraction of Class III YSOs in L1630 N predicted by our data of $\sim 15 \%$. This is still lower than expected on the basis of a cluster age of 1-2 Myr.

The surveys for PMS objects by Flaherty \& Muzerolle (2008) and Fang et al. (2009) produced a similar result of the high fraction of YSOs with disks and envelopes. Their surveys are rather complete in both space and flux and are based on different selection criteria, i.e., location in an optical/near-IR colormagnitude diagram with respect to the expected position of the main sequence at the cluster distance and subsequent spectroscopic follow up. Flaherty \& Muzerolle (2008) report a fraction of strong disk (Class I/II) of $66 \%$ and a fraction of MIPS-weak disks of $16 \%$, in perfect agreement with our fraction of disk objects after applying the correction of missed Class III sources. They also find a fraction of IRAC-weak disks (Class III) of $20 \pm 8 \%$, higher than our estimate but still lower than the fraction of Class III YSOs found in regions of similar age. Similarly, Fang et al. (2009) report a high fraction $(\sim 80 \%)$ of disks in the region, although this value might also be biased by their selection, which preferentially selects disk bearing young stars.

Alternatively, the substantial number of objects in younger SED classes is due to still ongoing star formation in L1630 N, and correspondingly young age $(\leq 1 \mathrm{Myr})$ for the studied YSO samples. As we will see in Sect. 7.3, some studies (Lada et al. 2010; Heiderman et al. 2010) have revealed that, if most of the present-day mass measured for a given cloud lies below a certain gas surface density threshold, which was determined by Heiderman et al. (2010) to $\sim 129 M_{\odot} \mathrm{pc}^{-2}$ (corresponding to $A_{\mathrm{V}} \approx 8.6 \mathrm{mag}$ ), a decrease in star formation could plausibly be caused by exhaustion of gas above such a threshold in surface density. Spezzi et al. (2011) demonstrated that this is the case for some clouds in the Lupus complex (Lup V and VI), where only $\sim 1 \%$ of the cloud mass lies above the threshold and, consistently, older SED classes (Class III) dominate the YSO population, while other Lupus clouds with 5 to $25 \%$ of the cloud mass above the threshold are mainly populated by younger SED classes (Class I to II). The fraction of cloud mass above the threshold in L1630 $\mathrm{N}$ is $\sim 35 \%$ (Table 4), i.e., even higher than in the most active star-forming region of Lupus (Lupus III), and may explain its exceptionally high disk fraction.

Thus, although the actual value might be slightly lower, the result of the high disk fraction in L1630 N seems to be real. The average disk fraction vs. age trend reported in the literature (e.g., Fedele et al. 2010) suggests a median disk lifetime around 2-3 Myr, meaning that $\sim 50 \%$ of the stars in a given population should have lost signatures of their disks after this time. However, several cases of clear outliers with respect to the average disk fraction vs. age trend have been reported in the literature. For example, Alcalá et al. (2008) found that only about
$20-30 \%$ of YSOs in Chamaeleon II have lost their primordial disks in about 4 Myr (i.e., the average age for its members), and Sicilia-Aguilar et al. (2006, 2013) measured a disk fraction of $\gtrsim 50 \%$ in the coeval cluster Trumpler 37 . Moreover, studies in NGC 3603 (Beccari et al. 2010) and the Magellanic Clouds (Spezzi et al. 2012; De Marchi et al. 2011a,b) indicate that a considerable fraction of PMS stars still exhibit signatures of accretion from a circumstellar disk at ages as old as 10 Myr. It is still under debate whether these differences from region to region are due to residual incompleteness effects of different surveys, limitations of the adopted selection methods, etc., or to the specific properties of the given star-forming environment (such as metallicity, presence of strong UV radiation fields, multiplicity, crowding, etc.), which may strongly affect disk evolution (e.g., Hollenbach et al. 2000; Linsky et al. 2007; Dullemond \& Dominik 2005; Johansen et al. 2009; Daemgen et al. 2013).

Evans et al. (2009) derived the half-life for each of the Lada classes from the combined analysis of the Spitzer c2d data set. According to this study, the half-life for Class II sources is $\sim 2$ Myr. If star formation has been continuous over a period longer than the age of Class II sources, the lifetime for each phase can be estimated by taking the ratio of number counts in each class with respect to Class II counts and multiplying by the lifetime for Class II. According to the statistics of Lada classes in L1630 N, we estimate a lifetime of 0.4 and $0.48 \mathrm{Myr}$ for the Class I and flat-spectrum phase, respectively (Table 2). These values agree with the lifetimes derived by Evans et al. (2009) by averaging all c2d clouds.

\section{Spatial distribution and clustering}

The spatial distribution of the different classes of objects in L1630 N is shown in Fig. 1 over-plotted on the VISTA extinction map, derived as explained in Sect. 7.1. The Class I and flat sources coincide or are located close to the sites of highest extinction, as observed in all young clusters still associated with the residual parental clouds.

NGC 2068 and NGC 2071 are the most prominent starforming clusters in the L $1630 \mathrm{~N}$ molecular cloud. The VISTA extinction map consistently shows two extinction peaks corresponding to the approximate center of NGC 2068 (RA $\approx$ $86.65 \mathrm{deg}, \mathrm{Dec} \approx 0.1 \mathrm{deg}$ ) and NGC 2071 (RA $\approx 86.75 \mathrm{deg}$, Dec $\approx 0.35 \mathrm{deg}$ ). It is also evident that, beside these two peaks, there is an additional extinction peak centered at $\mathrm{RA} \approx 86.55 \mathrm{deg}$ and Dec $\approx-0.15 \mathrm{deg}$. This third peak corresponds to the HH 2426 group of Herbig-Haro objects, and is very close to V1647 Ori (see Fig. 7 by Gibb 2008), a low-luminosity protostar, perhaps in a transition phase from Class I to Class II, which underwent a strong outburst in 2004 (Briceño et al. 2004). We also observe a very clear concentration of Class I and flat sources around this peak, confirming that the region is one of the several small centers of star formation in L1630. Another argument for still active, ongoing star formation is a notable concentration of presumably very young protostars in the region (see Stutz et al. 2013).

Lada \& Lada (2003) suggested that a cluster should be a group of some 35 members with a total mass density larger than $1 M_{\odot} \mathrm{pc}^{-3}$. To compare with this criterion and to assess the subclustering structures in L 1630, it is important to examine the distribution of YSOc in a quantitative and uniform way. We calculated the volume density of YSOs, based on their Lada classes and position, using a nearest neighbor algorithm similar to that applied by Gutermuth et al. (2005) and implemented by Jørgensen et al. (2008) for the c2d clouds. The calculations assume that the distribution of sources is locally spherical. We 
Table 3. Results of the clustering analysis and properties of star formation in substructures in Lynds $1630 \mathrm{~N}$.

\begin{tabular}{|c|c|c|c|c|c|c|c|c|c|c|c|}
\hline \multirow[t]{2}{*}{ Region } & \multirow[t]{2}{*}{ \#YSOs } & \multicolumn{4}{|c|}{ \# IR Class } & \multirow{2}{*}{$\begin{array}{l}\text { Mass } \\
\left(M_{\odot}\right)\end{array}$} & \multirow{2}{*}{$\begin{array}{l}\left\langle A_{\mathrm{V}}\right\rangle \\
(\mathrm{mag})\end{array}$} & \multirow{2}{*}{$\begin{array}{l}\text { SFE } \\
\%\end{array}$} & \multirow{2}{*}{$\begin{array}{l}\text { Area } \\
\left(\mathrm{pc}^{2}\right)\end{array}$} & \multirow{2}{*}{$\begin{array}{c}\text { Mass/Area }\left(\sum_{\text {gas }}\right) \\
\left(M_{\odot} \mathrm{pc}^{-2}\right)\end{array}$} & \multirow{2}{*}{$\begin{array}{l}S F R / \operatorname{Area}\left(\Sigma_{\mathrm{SFR}}\right) \\
\left(M_{\odot} \mathrm{Myr}^{-1} \mathrm{pc}^{-2}\right)\end{array}$} \\
\hline & & I & Flat & II & III & & & & & & \\
\hline Extended & 15 & 4 & 3 & 6 & 2 & 1840 & 0.3 & 0.4 & & $\ldots$ & $\ldots$ \\
\hline Lynds $1630 \mathrm{~N}$ & 171 & 21 & 27 & 120 & 3 & 2050 & 4.8 & 4.0 & 20.2 & 102 & 2.0 \\
\hline ...NGC 2071 & 52 & 5 & 12 & 34 & 1 & 400 & 8.8 & 3.8 & 2.17 & 180 & 6.0 \\
\hline ...NGC 2068 & 45 & 4 & 5 & 36 & 0 & 243 & 5.5 & 5.3 & 2.12 & 115 & 5.4 \\
\hline ...HH24-26 & 14 & 6 & 2 & 5 & 1 & 124 & 7.7 & 3.3 & 0.77 & 161 & 4.6 \\
\hline
\end{tabular}

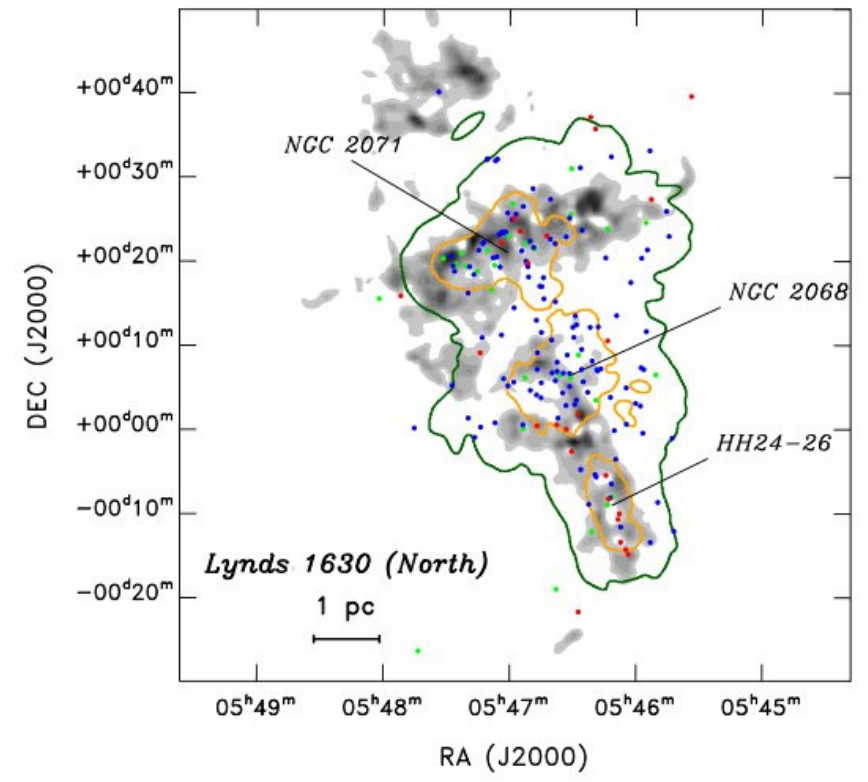

Fig. 8. Distribution of young stellar objects plotted over the extinction map (in gray) of the region. The green and orange contours correspond to volume densities of $1 M_{\odot} \mathrm{pc}^{-3}$ and $10 M_{\odot} \mathrm{pc}^{-3}$, respectively. Class I sources are shown in red, flat spectrum sources in green, and Class II sources in blue.

applied the algorithm to the whole sample of 186 YSOs in Table A.2. The overall results, reported in Table 3, are shown in Fig. 8.

To define a cluster, the $\mathrm{c} 2 \mathrm{~d}$ surveys adopted the tighter level of 25 times the Lada \& Lada (2003) criterion (i.e., a mass density of $25 M_{\odot} \mathrm{pc}^{-3}$ ), which normally provides the already established cluster and group boundaries. Within the c2d surveys, "clusters" are regions with more than 35 YSOs within a given volume density contour and "groups" are regions with less. Five is the lowest number of YSOs considered to constitute a separate entity. "Clusters" and "groups" can be either "tight" or "loose" depending on whether their volume densities, $\rho$, are higher than 25 or $1 M_{\odot} \mathrm{pc}^{-3}$ (corresponding to 50 and 2 YSOs $\mathrm{pc}^{-3}$ ), respectively, assuming an average YSO mass of $0.5 M_{\odot}$. As noted in the c2d papers, these criteria are useful as a way of making a direct comparison between regions within clouds and across different clouds, but being empirical they should not be used as evidence for discussions on whether the star formation process is hierarchical or not.

In L1630 N we identify structures with two levels of volume densities, structures with a density larger than $1 M_{\odot} \mathrm{pc}^{-3}$ (similar to the criterion for a cluster by Lada \& Lada 2003) or structures with a density as high as $10 M_{\odot} \mathrm{pc}^{-3}$. Note, however, the latter is lower than what was applied for "tight" associations in the c2d surveys. Thus, according to the $\mathrm{c} 2 \mathrm{~d}$ criteria, the loose clusters identified in this region are $\mathrm{L} 1630 \mathrm{~N}$ as a whole with a density of $1 M_{\odot} \mathrm{pc}^{-3}$ and NGC 2071 and NGC 2068 with a density of
Table 4. Overall properties of the star formation in L1630 N.

\begin{tabular}{|c|c|c|c|}
\hline Property & Value & Uncertainty range & Unit \\
\hline Peak of the KLM & 0.5 & $0.3-0.7$ & $\overline{M_{\odot}}$ \\
\hline Cloud area $\left(A_{\mathrm{V}} \geq 2 \mathrm{mag}\right)$ & 39 & - & $\mathrm{pc}^{2}$ \\
\hline Cloud mass ( $\left.A_{\mathrm{V}} \geq 2 \mathrm{mag}\right)$ & 3865 & $3200-4600$ & $M_{\odot}$ \\
\hline Cloud density $\left(\Sigma_{\mathrm{gas}}\right)$ & 98 & $81-116$ & $M_{\odot} \mathrm{pc}^{-2}$ \\
\hline Fraction of cloud above $\Sigma_{\text {th }}$ & 0.35 & - & \\
\hline N. YSOc/Area & 5 & - & $\mathrm{pc}^{-2}$ \\
\hline$S F E$ & 2.35 & $2-2.8$ & percent \\
\hline$S F R$ & 75 & $47-103$ & $M_{\odot} / \mathrm{Myr}$ \\
\hline$S F R /$ area $\left(\Sigma_{\mathrm{SFR}}\right)$ & 1.9 & $1.2-2.6$ & $M_{\odot} \mathrm{Myr}^{-1} \mathrm{pc}^{-2}$ \\
\hline
\end{tabular}

$10 M_{\odot} \mathrm{pc}^{-3}$. The HH24-26 entity can be defined as a loose group with a density of $10 M_{\odot} \mathrm{pc}^{-3}$.

\section{Overall results on star formation in $\mathrm{L} 1630 \mathrm{~N}$}

In this section, we analyze and discuss the cloud properties of $\mathrm{L} 1630 \mathrm{~N}$ and the global properties of star formation in this region on the basis of the VISTA observations and our YSOc sample. Our results are summarized in Table 4.

\subsection{Extinction map, cloud mass, and surface density}

An extinction map, with a resolution of $30^{\prime \prime}$, was constructed for the entire area of Orion observed in the VISTA SV mini-survey (Lombardi et al., in prep.). The technique used is optimized to produce highly accurate extinction maps from multiband nearIR photometric data as outlined in Lombardi \& Alves (2001, NICER) and Lombardi (2009, NICEST). The method is the natural generalization of the near-infrared color excess (NICE) method of Lada et al. (1994) and produces significantly less noisy and, hence, more accurate extinction maps taking advantage of all bands available. Applications of this technique to 2MASS data has shown an improvement with respect to the standard NICE algorithm by a factor 2 on the noise variance (Lombardi \& Alves 2001). We further compare our extinction map values with the spectroscopic values inferred by Flaherty \& Muzerolle (2008) for a sample of 67 previously known PMS stars in the cloud and found a good agreement (within 23 visual mag), but also an apparent shift of $A_{\mathrm{V}}=3.4$ mag (i.e., the extinction map gave a consistently higher $A_{\mathrm{V}}$ than what was determined from spectroscopy). This has been observed for other regions as well and can be explained by the fact that the extinction map measures the extinction through the whole cloud, while the PMS stars are located in the cloud.

According to this extinction map, the extinction in the L1630 N cloud (tile No. 12) is typically low ( $A_{V} \approx 1 \mathrm{mag}$ ), with peaks up to $A_{\mathrm{V}} \approx 20 \mathrm{mag}$ occurring close to the following locations: $\mathrm{RA}=86.75 \mathrm{deg}$ and $\mathrm{Dec}=0.35 \mathrm{deg}$ (i.e., the center of NGC 2071), RA $=86.65 \mathrm{deg}$ and $\mathrm{Dec}=0.1 \mathrm{deg}$ (i.e., the center of NGC 2068) and RA $=86.55 \mathrm{deg}$ and $\mathrm{Dec}=-0.15$, in which we identified a group of YSOc (Sect. 6). 
Using the extinction map and assuming a distance of $400 \mathrm{pc}$, we also estimated the cloud mass for the L1630 N complex. To this aim, we used the relationship between gas surface density, $\Sigma_{\text {gas }}$, and extinction by Heiderman et al. (2010), i.e., $\Sigma_{\text {gas }}=15 \times \mathrm{A}_{\mathrm{V}} M_{\odot} / \mathrm{pc}^{2}$. We estimated that the total cloud mass for $A_{\mathrm{V}} \geq 2 \mathrm{mag}$ is about $3865 M_{\odot}{ }^{7}$; considering that the area where $A_{\mathrm{V}} \geq 2$ mag extends over $\sim 39 \mathrm{pc}^{2}$, the cloud column density is about $\sim 100 M_{\odot} \mathrm{pc}^{-2}$. The NICE and NICER methods provide an intrinsic error of about $0.5 \mathrm{mag}$, on the average. Assuming this value as the intrinsic error of our VISTA extinction map, and an error on the distance to the cloud of about $10 \%$ (see Sect. 2 in Gibb 2008), we estimated that the cloud mass and column density of L $1630 \mathrm{~N}$ can be safely placed in the ranges $3200-4600 M_{\odot}$ and 81-116 $M_{\odot} \mathrm{pc}^{-2}$, respectively.

We stress that in $\sim 35 \%$ of the cloud area where $A_{\mathrm{V}} \geq 2$ mag, the extinction is above $8.6 \mathrm{mag}$. As concluded in Heiderman et al. (2010) the latter value for the extinction sets an important threshold at which the gas surface density is linearly proportional to the surface density of the star formation rate.

\subsection{Star formation efficiency}

We derive the global star formation efficiency (SFE) in $\mathrm{L} 1630 \mathrm{~N}$ as

$S F E=\frac{M_{\text {stars }}}{M_{\text {cloud }}+M_{\text {stars }}}$,

where $M_{\text {cloud }}$ is the cloud mass derived in Sect. 7.1, and $M_{\text {star }}$ is the total mass converted into stars. The parameter $M_{\text {star }}$ is derived from the number of YSOc identified in our study, i.e., without applying any corrections for missed diskless star, following the procedure applied by the Spitzer-c2d/GB surveys. As already pointed out by Evans et al. (2009), this implies that the star formation efficiencies and rates over the whole lifetime of a starforming cloud could be higher. For L1630 N, we estimated $M_{\text {star }}$ to be $\sim 93 M_{\odot}$, assuming an average YSO mass of $0.5 M_{\odot}$ consistent with the peak observed in the KLF (Fig. 6), and with the assumption made in all clouds observed by the Spitzer-c2d/GB surveys, which we use for comparison.

We find that the overall SFE in L1630 N ranges between $2 \%$ and $2.8 \%$, and is $\sim 4.0 \%$ on average for the L1630 N clusters (Table 3), which is consistent overall with the typical values measured for Orion A and B (Federrath \& Klessen 2013, and references therein) for all c2d clouds (see Table 4 by Evans et al. 2009), and, in general, for the majority of star-forming regions in the Galaxy (e.g., Federrath \& Klessen 2013). However, we notice that the SFE in L1630 N is lower than measured in the Orion bright-rimmed clouds (e.g., 5 to $10 \%$; Lee et al. 2005) and, in particular, lower than measured in subclusters in the southern region L1630 S. For the populous cluster NGC 2024, Lada et al. (1997) determined a high SFE of $~ 30 \%$, although based on CS observations that trace only the highest density gas, and hence providing a lower cloud mass than the mass we obtained via the extinction map method. It appears that the CS measurements underestimate the total cloud mass by a factor of 3-4 as compared to the cloud mass derived from the extinction map, such that the true SFE for NGC 2024 is likely more like $\sim 10 \%$. In comparison, the SFE for the subclusters identified in L1630 N is very low (see Table 3). We recall that it is expected that star formation may be more efficient in localized, compressed regions, where triggered star formation might play a role, but perhaps not so in the entire cloud (Lee et al. 2005). The different

\footnotetext{
7 Note that the total mass of gas in dense cores in L1630 $\mathrm{N}$ has been estimated to be $\sim 2000 M_{\odot}$ (see Sect. 3.1 in Gibb 2008).
}

SFE measured between the northern and the southern regions of L1630 might indicate that two different star formation mechanisms currently dominate in the two regions of this cloud.

\subsection{Star formation rate and star formation density}

We derive the star formation rate (SFR) in $\mathrm{L} 1630 \mathrm{~N}$ as

$S F R=\frac{M_{\text {stars }}}{\text { Age }}$

where $M_{\text {star }}$ is the total mass converted into stars (equal to $\sim 93 M_{\odot}$, Sect. 7.2) and Age is the average age of the YSO population. The latter has some uncertainty and therefore determines the possible range for the resulting SFR. A median age of $2 \mathrm{Myr}$ was determined by Flaherty \& Muzerolle (2008) for their optical spectroscopy sample in NGC 2068/71. However, this could be an overestimate for the L1630 N YSO sample in our work, which does include the large majority of the FM08 objects but also include highly embedded, i.e. potentially much younger, sources. Note that Fang et al. (2009) estimate a median age of $0.9 \mathrm{Myr}$ for their YSO sample of L1630 N, of which $60 \%$ are included in our survey.

Taking the age uncertainty into account, we find that the L1630 N cloud is turning some $75 \pm 28 M_{\odot}$ into YSOs every Myr. This SFR is in agreement with the SFR measured for the c2d/GB clouds (see Table 3 by Evans et al. 2009), with the exception of Cha II, where the SFR seems to be very low (Alcalá et al. 2008). However, the SFR in L1630 N appears lower than the average SFR measured for the overall Orion A and B molecular clouds (150-700 $M_{\odot} / \mathrm{Myr}$; see Table 2 by Lada et al. 2010) and the local SFR measured for the ONC (Lada et al. 1996) and Trapezium (Palla \& Stahler 1999; Lada \& Lada 2003). We note, however, that large SFR variations are observed among the Orion subregions; for example, the SFR in L1630 (to which L1630 N belongs) is known to be a factor of 2 to 7 lower than observed in the nearby L1641 (Meyer et al. 2008).

These variations can be reconciled if instead we consider the SFR per unit area $\left(\Sigma_{\mathrm{SFR}}\right)$, i.e., the density of star formation. It has been confirmed that $\Sigma_{\mathrm{SFR}}$ is linearly proportional to the cloud gas surface density $\left(\Sigma_{\text {gas }}\right)$, above an extinction threshold of $A_{\mathrm{V}} \approx 8.6 \mathrm{mag}$ (Heiderman et al. 2010), corresponding to a gas density threshold $\left(\Sigma_{\text {th }}\right)$ of $\sim 129 M_{\odot} \mathrm{pc}^{-2}$. We measure for $\mathrm{L} 1630 \mathrm{~N}$ a $\Sigma_{\mathrm{SFR}}$ of $1.9 \mathrm{M}_{\odot} \mathrm{Myr}^{-1} \mathrm{pc}^{-2}$ and $\Sigma_{\text {gas }}$ of $\sim 98 \mathrm{M}_{\odot} \mathrm{pc}^{-2}$, and these values are in excellent agreement with previous observations of Galactic star-forming activity (Heiderman et al. 2010). Note also that, as mentioned in the previous section, about $35 \%$ of the cloud has $A_{\mathrm{V}}>8.6$ mag. Thus, more than one-third of the cloud has a $\Sigma_{\text {gas }}$ above $\Sigma_{\text {th }}$. At the level of the substructures identified in the clustering analysis, the values of $\Sigma_{\mathrm{SFR}}$ and $\Sigma_{\text {gas }}$ are slightly higher (see Table 3 ) but still well within the range for Galactic star-forming regions (Heiderman et al. 2010).

The linear correlation between the rate of star formation and the amount of dense gas in molecular clouds lies above the extragalactic SFR-gas relations (e.g., Kennicutt-Schmidt law; Kennicutt 1998) up to a factor of 17 to 54 (Heiderman et al. 2010). This correlation is confirmed by all c2d/GB clouds (Heiderman et al. 2010), all nearby molecular clouds (Lada et al. 2010), Galactic massive dense clumps (Wu et al. 2010), the youngest and still embedded Class I and flat-spectrum YSOs in the Galaxy (Heiderman et al. 2010), and is also consistent with the results for several nearby molecular clouds (Gutermuth et al. 2011). Moreover, the extragalactic SFR-gas relation is not linear, because $\Sigma_{\mathrm{SFR}}$ scales as $\Sigma_{\text {gas }}^{1.4}$. Several contributing factors 
to this difference have been identified so far (Heiderman et al. 2010): i) much of $\Sigma_{\text {gas }}$ is below $\Sigma_{\text {th }}$ in extragalactic studies, which average over large scales and include both star-forming gas and gas that is not dense enough to form stars; and ii) using ${ }^{12} \mathrm{CO}$ or ${ }^{13} \mathrm{CO}$ to measure the $\mathrm{H}_{2}$ in galaxies gives systematically lower $\Sigma_{\text {gas }}$ than Galactic $A_{\mathrm{V}}$ measurements, as the one we used, by a factor up to $30 \%$. Indeed, power-law indices between 0.8 and 1.6 have been found for the extragalactic SFR-gas relation (e.g., Kennicutt et al. 2007; Bigiel et al. 2008; Krumholz et al. 2009), depending on the survey spatial resolution and the adopted tracer. These overall results suggest that the key to obtaining a predictive understanding of the star formation rates in molecular clouds and galaxies is to understand those physical factors that give rise to the dense components of these clouds (Lada et al. 2010).

\section{Summary and conclusions}

Based on the VISTA Orion mini-survey, complemented with Spitzer observations, we performed a study of the YSO population and star formation in the L1630 $\mathrm{N}$ cloud. The c2d multicolor criteria selected 186 YSOs in the area of about 1.5 sq. deg in $\mathrm{L} 1630 \mathrm{~N}$. The census is $\sim 95 \%$ complete down to $M_{\star} \sim$ $0.02 M_{\odot}$. We investigated both the YSOs with infrared excess selected according to the c2d criteria, as well as the other YSOs cloud members and candidates from the previous surveys. Spectroscopic follow ups, published in the literature, confirm the YSO nature of most of the selected candidates, supporting the reliability of the selection criteria.

The $K$-band luminosity function of $\mathrm{L} 1630 \mathrm{~N}$ shows a broad peak between 10.5 and $12 \mathrm{mag}$, i.e. $0.3-0.7 M_{\odot}$, but steadily declines to the hydrogen-burning limit at $K_{\mathrm{S}} \approx 13 \mathrm{mag}$. We predict a fraction of $28 \%$ young substellar objects, but we note that the expected contamination from field stars mimicking the colors of BDs is on the order of $50 \%$, while in the stellar regime it is about $20 \%$. Thus, the actual fraction of substellar objects in L1630 N may be $\sim 20 \%$. The $K$-band luminosity function of $\mathrm{L} 1630 \mathrm{~N}$ is remarkably similar to that of the Trapezium cluster.

The analysis of the SEDs shape shows that the L1630 N population is dominated by objects with active accretion, with only a minority being systems with passive disks. The disk/envelope fraction in the region of $\sim 85 \%$ is high in comparison with other star formation regions of similar age. The fraction of the Class I and flat-spectrum sources ( $13 \%$ and $16 \%$, respectively) in $\mathrm{L} 1630 \mathrm{~N}$ and their respective phase lifetime $(0.4$ and $0.48 \mathrm{Myr}$, respectively) are consistent with the results for the $\mathrm{c} 2 \mathrm{~d}$ clouds (Evans et al. 2009).

We studied the spatial distribution and volume density of the 186 YSOs with the following results: we identify structures with volume densities higher than $1 M_{\odot} \mathrm{pc}^{-3}$ or $10 M_{\odot} \mathrm{pc}^{-3}$. The loose clusters identified are $\mathrm{L} 1630 \mathrm{~N}$ as a whole, with a density of $1 M_{\odot} \mathrm{pc}^{-3}$, and NGC 2071 and NGC 2068, with a density of $10 M_{\odot} \mathrm{pc}^{-3}$. The HH24-26 entity can be defined as a loose group with a density of $10 M_{\odot} \mathrm{pc}^{-3}$.

The cloud mass determined from the VISTA extinction map is on the order of $3865 M_{\odot}$ and the SFE of $2-2.8 \%$ is similar to previous estimates for the Orion A and B clouds and for the c2d clouds, but is much lower than the SFE measured in subclusters in the southern region L1630 S. The SFE of the subclusters in L $1630 \mathrm{~N}$ is also comparably low. The different SFE in the northern and southern regions of L1630 might suggest different star formation mechanisms. The SFR is similar to that of the c2d clouds (Evans et al. 2009); we find that L1630 N is turning some $75 M_{\odot}$ into YSOs every Myr. This is, however, lower than the average value measured for the Orion A and B clouds and the local SFR for the ONC and the Trapezium, but large variations of the SFR among the Orion subgroupings are observed. These variations disappear when considering the density of star formation $\Sigma_{\mathrm{SFR}}$. The density of star formation $\sim 2 \mathrm{M}_{\odot} \mathrm{Myr}^{-1} \mathrm{pc}^{-2}$ and the gas surface density $\sim 98 M_{\odot} \mathrm{pc}^{-2}$ in $\mathrm{L} 1630 \mathrm{~N}$ are in excellent agreement with previous determinations of Galactic starforming activity. At the level of the subclusters in L1630 N, these quantities are also similar to those in the subclusters in other Galactic star-forming regions. More than one-third of the cloud in $\mathrm{L} 1630 \mathrm{~N}$ has a gas surface density above $\Sigma_{\mathrm{th}} \sim 129 \mathrm{M}_{\odot} \mathrm{pc}^{-2}$. This may indicate that star formation in L1630 N is still ongoing, which may explain the exceptionally high disk/envelope fraction in the region. The latter, however, needs to be confirmed in the future with deep observations tracing the complete population of young diskless sources.

Acknowledgements. We thank the anonymous referee for valuable comments that further improved the clarity of the paper. J.M.A. acknowledges financial support from INAF under the program PRIN2013 "Disk jets and the dawn of planets". This research has made use of the SIMBAD database operated at CDS, Strasbourg, France. It also makes use of data products from the Two Micron All Sky Survey, which is a joint project of the University of Massachusetts and the Infrared Processing and Analysis Center/California Institute of Technology, funded by the National Aeronautics and Space Administration and the National Science Foundation. We greatly appreciate the work done by the UK-based VISTA consortium who built and commissioned the VISTA telescope and camera.

\section{Appendix A: Theoretical isochrones in the VISTA photometric system}

Theoretical isochrones for low-mass stars and BDs down to $0.001 M_{\odot}$ are provided by Baraffe et al. (1998) and Chabrier et al. (2000) in the Cousins photometric system (Bessell 1990), and are the most commonly used for very low-mass stellar population studies. In particular, they are extensively used to select PMS star and young BD candidates on the basis of color-magnitude diagrams (CMDs). Since the transmission curves of the VISTA filters are very different from the Cousins filter transmission curves, we transformed these isochrones into the specific VISTA photometric system. In this way, we make available to the community a valuable tool to be used in extensive VISTA-based searches for very low-mass stars and BDs in other star-forming regions.

The procedure adopted to perform the conversion of the evolutionary models from one system to another has been already described in detail in Spezzi et al. (2007, Appendix B). The expected flux $\left(f_{\Delta \lambda}\right)$ at the stellar surface in the VISTA pass bands were determined by integrating the synthetic lowresolution spectra for low-mass stars by Hauschildt et al. (1999), calculated with their NextGen model-atmosphere code, under the filter transmission curves $^{8}$ (see Fig. A.1, upper panel). To simulate very cool objects (i.e. $T_{\text {eff }}<3000 \mathrm{~K}$ ), we used the AMES-Dusty and AMES-Cond atmosphere models by Allard et al. (2001), which take the formation of condensed species significantly modifying the atmospheric structure ${ }^{9}$ into account. The expected flux $f_{\Delta \lambda}$ was then converted to absolute magnitudes

\footnotetext{
8 Available at http://apm49.ast.cam.ac.uk/ surveys-projects/vista/technical/filter-set

9 While in the AMES-Dusty models the condensed species are included both in the equation of state and in the opacity, taking dust scattering and absorption into account, in the AMES-Cond models the opacity of these condensates is ignored to mimic a rapid gravitational settling of all grains below the photosphere.
} 

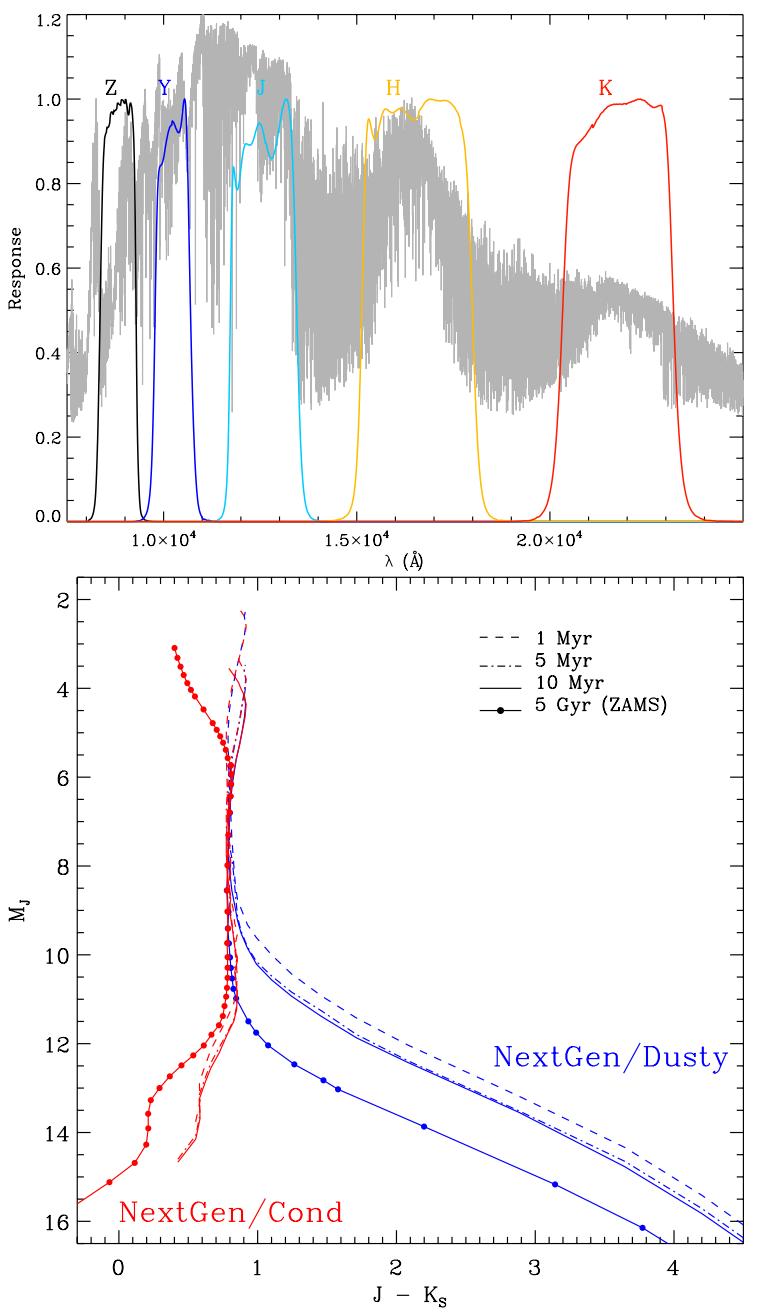

Fig. A.1. Upper panel: the $Z Y J H K_{\mathrm{S}}$ VISTA transmission bands. An example of a normalized NextGen spectrum (Hauschildt et al. 1999) with $T_{\text {eff }}=2500 \mathrm{~K}$ is overplotted. Lower panel: theoretical $M_{J}$ vs. $J-K_{\mathrm{S}}$ diagram. The isochrones, shifted to the distance of 10 pc (i.e., absolute magnitudes), are in the VISTA photometric system. Different curve styles correspond to different ages, as indicated in the legend. We plot in blue the isochrones computed using the NextGen/AMES-Dusty atmospheric models, and in red those computed using the NextGen/AMESCond models.

using the following equation:

$m_{\Delta \lambda}=-2.5 \cdot \log _{10}\left(f_{\Delta \lambda} \cdot \frac{R_{\star}^{2}}{d^{2}}\right)+C_{\Delta \lambda}$,

where $d=10 \mathrm{pc}, R_{\star}$ is the stellar radius expected for PMS objects and computed from the theoretical PMS evolutionary tracks by Baraffe et al. (1998) for low-mass stars and those by Chabrier et al. (2000) for substellar objects (i.e., $M \lesssim 0.1 M_{\odot}$ ), and $C_{\Delta \lambda}$ is the absolute calibration constant of the VISTA photometric system, tied to the Earth flux of an A0-type star with magnitude $V=0{ }^{10}$. In Fig. A.1 (lower panel) we show, as an example, the theoretical 1, 5, and $10 \mathrm{Myr}$ isochrones and the ZAMS (5 Gyr) on the $M_{J}$ vs. $J-K_{\mathrm{S}} \mathrm{CMD}$ and make them publicly available in Table A.1, where we also give isochrones for $50 \mathrm{Myr}$ and $100 \mathrm{Myr}$.

\footnotetext{
${ }^{10}$ http://apm49.ast.cam.ac.uk/surveys-projects/vista/ technical/photometric-properties
}

\section{References}

Alcalá, J. M., Spezzi, L., Chapman, N., et al. 2008, ApJ, 676, 427

Allard, F., Hauschildt, P. H., Alexander, D. R., Tamanai, A., \& Schweitzer, A. 2001, ApJ, 556, 357

Arnaboldi, M., Petr-Gotzens, M., Rejkuba, M., et al. 2010, The Messenger, 139, 6

Baraffe, I., Chabrier, G., Allard, F., \& Hauschildt, P. H. 1998, A\&A, 337, 403 Basri, G., \& Reiners, A. 2006, AJ, 132, 663

Beccari, G., Spezzi, L., De Marchi, G., et al. 2010, ApJ, 720, 1108 Bessell, M. S. 1990, PASP, 102, 1181

Bigiel, F., Leroy, A., Walter, F., et al. 2008, AJ, 136, 2846

Briceño, C., Luhman, K. L., Hartmann, L., Stauffer, J. R., \& Kirkpatrick, J. D. 2002, ApJ, 580, 317

Briceño, C., Vivas, A. K., Hernández, J., et al. 2004, ApJ, 606, L123

Cardelli, J. A., Clayton, G. C., \& Mathis, J. S. 1989, ApJ, 345, 245

Chabrier, G., Baraffe, I., Allard, F., \& Hauschildt, P. 2000, ApJ, 542, 464

Cieza, L. A., Schreiber, M. R., Romero, G. A., et al. 2010, ApJ, 712, 925

Comerón, F. 2008, Handbook of Star Forming Regions, Vol. II, 295

Daemgen, S., Petr-Gotzens, M. G., Correia, S.., et al. 2013, A\&A, 554, A43

Dalton, G. B., Caldwell, M., Ward, A. K., et al. 2006, Proc. SPIE, 6269

De Marchi, G., Panagia, N., Romaniello, M., et al. 2011a, ApJ, 740, 11

De Marchi, G., Paresce, F., Panagia, N., et al. 2011b, ApJ, 739, 27

Dullemond, C. P., \& Dominik, C. 2005, A\&A, 434, 971

Dunham, M. M., Arce, H. G., Allen, L. E., et al. 2013, AJ, 145, 94

Duquennoy, A., \& Mayor, M. 1991, Bioastronomy: The Search for Extraterrestial Life - The Exploration Broadens, 390, 39

Emerson, J., McPherson, A., \& Sutherland, W. 2006, The Messenger, 126, 41

Evans, N. J., II 2008, Final Delivery of Data from the c2d Legacy Project: IRAC and MIPS (Pasadena: SSC)

Evans, N. J., II, Dunham, M. M., Jørgensen, J. K., et al. 2009, ApJS, 181, 321

Fang, M., van Boekel, R., Wang, W., et al. 2009, A\&A, 504, 461

Fazio, G. G., Hora, J. L., Allen, L. E., et al. 2004, ApJS, 154, 10

Fedele, D., van den Ancker, M. E., Henning, T., Jayawardhana, R., \& Oliveira, J. M. 2010, A\&A, 510, A72

Federrath, C., \& Klessen, R. S. 2013, ApJ, 763, 51

Flaherty, K. M., \& Muzerolle, J. 2008, AJ, 135, 966

Gibb, A. G. 2008, Handbook of Star Forming Regions, Vol. I, 693

Greene, T. P., Wilking, B. A., Andre, P., Young, E. T., \& Lada, C. J. 1994, ApJ, 434, 614

Gutermuth, R. A., Megeath, S. T., Pipher, J. L., et al. 2005, ApJ, 632, 397

Gutermuth, R. A., Pipher, J. L., Megeath, S. T., et al. 2011, ApJ, 739, 84

Haisch, K. E., Jr., Lada, E. A., \& Lada, C. J. 2001, ApJ, 553, L153

Harvey, P., Merín, B., Huard, T. L., et al. 2007a, ApJ, 663, 1149

Harvey, P. M., Rebull, L. M., Brooke, T., et al. 2007b, ApJ, 663, 1139

Hatchell, J., Terebey, S., Huard, T., et al. 2012, ApJ, 754, 104

Hauschildt, P. H., Allard, F., \& Baron, E. 1999, ApJ, 512, 377

Heiderman, A., Evans, N. J., II, Allen, L. E., Huard, T., \& Heyer, M. 2010, ApJ, 723,1019

Hollenbach, D. J., Yorke, H. W., \& Johnstone, D. 2000, Protostars and Planets IV, 401

Hsu, W.-H, Hartmann, L., Allen, L., et al. 2012, ApJ, 752, 59

Johansen, A., Youdin, A., \& Mac Low, M.-M. 2009, ApJ, 704, L75

Jørgensen, J. K., Johnstone, D., Kirk, H., et al. 2008, ApJ, 683, 822

Kennicutt, R. C., Jr. 1998, ApJ, 498, 541

Kennicutt, R. C., Jr., Calzetti, D., Walter, F., et al. 2007, ApJ, 671, 333

Krumholz, M. R., McKee, C. F., \& Tumlinson, J. 2009, ApJ, 693, 216

Irwin, M. J., Lewis, J., Hodgkins, S., et al. 2004, Proc. of SPIE, 5493, 411

Lada, C. J. 2006, ApJ, 640, L63

Lada, C. J., \& Lada, E. A. 2003, ARA\&A, 41, 57

Lada, C. J., \& Wilking, B. A. 1984, ApJ, 287, 610

Lada, C. J., Lada, E. A., Clemens, D. P., \& Bally, J. 1994, ApJ, 429, 694

Lada, E. A., Depoy, D. L., Evans, N. J., \& Gatley, I. 1991, ApJ, 371, 171

Lada, C. J., Alves, J., \& Lada, E. A. 1996, AJ, 111, 1964

Lada, E. A., Evans, N. J. II, \& Falgarone, E. 1997, ApJ, 488, 286

Lada, C. J., Lombardi, M., \& Alves, J. F. 2010, ApJ, 724, 687

Lee, H.-T., Chen, W. P., Zhang, Z.-W., \& Hu, J.-Y. 2005, ApJ, 624, 808

Linsky, J. L., Gagné, M., Mytyk, A., McCaughrean, M., \& Andersen, M. 2007, ApJ, 654, 347

Lombardi, M. 2009, A\&A, 493, 735

Lombardi, M., \& Alves, J. 2001, A\&A, 377, 1023

Lonsdale, C. J., Smith, H. E., Rowan-Robinson, M., et al. 2003, PASP, 115, 89

López Martí, B., Eislöffel, J., Scholz, A., \& Mundt, R. 2004, A\&A, 416, 555

Luhman, K. L., \& Rieke, G. H. 1999, ApJ, 525, 440

Mason, B. D., Henry, T. J., Hartkopf, W. I., ten Brummelaar, T., \& Soderblom, D. R. 1998, AJ, 116, 2975

Megeath, S. T., Gutermuth, R., Muzerolle, J., et al. 2012, AJ, 144, 192

Merín, B., Jørgensen, J., Spezzi, L., et al. 2008, ApJS, 177, 551

Meyer, M. R., Calvet, N., \& Hillenbrand, L. A. 1997, AJ, 114, 288 


\section{Spezzi et al.: The VISTA survey in L1630 N}

Meyer, M. R., Flaherty, K., Levine, J. L., et al. 2008, Handbook of Star Forming Regions, Vol. I, 662

Miesch, M. S., \& Bally, J. 1994, ApJ, 429, 645

Muench, A. A., Lada, E. A., Lada, C. J., \& Alves, J. 2002, ApJ, 573, 366

Oliveira, I., Merín, B., Pontoppidan, K. M., et al. 2009, ApJ, 691, 672

Palla, F., \& Stahler, S. W. 1999, ApJ, 525, 772

Petr-Gotzens, M., Alcalá, J. M., Briceño, C., et al. 2011, The Messenger, 145, 29

Principe D. A., Kastner, J. H., Grosso, N., et al. 2014, ApJS, 213, 4

Rieke, G. H., Young, E. T., Engelbracht, C. W., et al. 2004, ApJS, 154, 25

Robin, A. C., Reylé, C., Derrière, S., \& Picaud, S. 2003, A\&A, 409, 523

Santiago, B. X., Gilmore, G., \& Elson, R. A. W. 1996, MNRAS, 281, 871

Scholz, A., Muzic, K., Geers, V., et al. 2012, ApJ, 744, 6

Sicilia-Aguilar, A., Hartmann, L., Calvet, N., et al. 2006, ApJ, 638, 89

Sicilia-Aguilar, A., Kim, J. S., Sobolev, A., et al. 2013, A\&A, 559, A3

Skrutskie, M. F., Cutri, R. M., Stiening, R., et al. 2006, AJ, 131, 1163

Spezzi, L., Alcalá, J. M., Frasca, A., Covino, E., \& Gandolfi, D. 2007, A\&A, 470,281
Spezzi, L., Alcalá, J. M., Covino, E., et al. 2008, ApJ, 680, 1295

Spezzi, L., Pagano, I., Marino, G., et al. 2009, A\&A, 499, 541

Spezzi, L., Vernazza, P., Merín, B., et al. 2011, ApJ, 730, 65

Spezzi, L., de Marchi, G., Panagia, N., Sicilia-Aguilar, A., \& Ercolano, B. 2012, MNRAS, 421, 78

Strom, K. M., Strom, S. E., \& Merrill, K. M. 1993, ApJ, 412, 233

Stutz, A. M., Tobin, J. J., Stanke, Th., et al. 2013, AJ, 767, 36

Surace, J. A., Shupe, D. L., Fang, F., et al. 2004, VizieR Online Data Catalog: II/255

Wainscoat, R. J., Cohen, M., Volk, K., Walker, H. J., \& Schwartz, D. E. 1992, ApJS, 83, 111

Watson, M. G., Schröder, A. C., Fyfe, D., et al. 2009, A\&A, 493, 339

White, R. J., \& Basri, G. 2003, ApJ, 582, 1109

Whitworth, A., Bate, M. R., Nordlund, Å., Reipurth, B., \& Zinnecker, H. 2007, Protostars and Planets V, 459

Wu, J., Evans, N. J., II, Shirley, Y. L., \& Knez, C. 2010, ApJS, 188, 313

Zacharias, N., Finch, C. T., Girard, T. M., et al. 2013, AJ, 145, 44 